\title{
Asymptotic theory of resonant flow in a spheroidal cavity driven by latitudinal libration
}

\author{
Keke Zhang ${ }^{1,2} \dagger$, Kit H. Chan ${ }^{3}$ and Xinhao Liao $^{4}$ \\ ${ }^{1}$ Department of Mathematical Sciences, University of Exeter, Exeter EX4 4QF, UK \\ ${ }^{2}$ Institute of Mathematical Sciences, Chinese University of Hong Kong, Shatin, Hong Kong \\ ${ }^{3}$ Department of Mathematics, University of Hong Kong, Pokfulam, Hong Kong \\ ${ }^{4}$ Shanghai Astronomical Observatory, Chinese Academy of Sciences, Shanghai 200030, China
}

(Received 17 May 2011; revised 28 October 2011; accepted 24 November 2011; first published online 6 January 2012)

We consider a homogeneous fluid of viscosity $v$ confined within an oblate spheroidal cavity, $x^{2} / a^{2}+y^{2} / a^{2}+z^{2} /\left(a^{2}\left(1-\mathscr{E}^{2}\right)\right)=1$, with eccentricity $0<\mathscr{E}<1$. The spheroidal container rotates rapidly with an angular velocity $\boldsymbol{\Omega}_{0}$, which is fixed in an inertial frame and defines a small Ekman number $E=v /\left(a^{2}\left|\Omega_{0}\right|\right)$, and undergoes weak latitudinal libration with frequency $\hat{\omega}\left|\Omega_{0}\right|$ and amplitude $P o\left|\Omega_{0}\right|$, where $P o$ is the Poincaré number quantifying the strength of Poincaré force resulting from latitudinal libration. We investigate, via both asymptotic and numerical analysis, fluid motion in the spheroidal cavity driven by latitudinal libration. When $\left|\hat{\omega}-2 /\left(2-\mathscr{E}^{2}\right)\right| \gg O\left(E^{1 / 2}\right)$, an asymptotic solution for $E \ll 1$ and $P o \ll 1$ in oblate spheroidal coordinates satisfying the no-slip boundary condition is derived for a spheroidal cavity of arbitrary eccentricity without making any prior assumptions about the spatial-temporal structure of the librating flow. In this case, the librationally driven flow is nonaxisymmetric with amplitude $O(P o)$, and the role of the viscous boundary layer is primarily passive such that the flow satisfies the no-slip boundary condition. When $\left|\hat{\omega}-2 /\left(2-\mathscr{E}^{2}\right)\right| \ll O\left(E^{1 / 2}\right)$, the librationally driven flow is also non-axisymmetric but latitudinal libration resonates with a spheroidal inertial mode that is in the form of an azimuthally travelling wave in the retrograde direction. The amplitude of the flow becomes $O\left(P o / E^{1 / 2}\right)$ at $E \ll 1$ and the role of the viscous boundary layer becomes active in determining the key property of the flow. An asymptotic solution for $E \ll 1$ describing the librationally resonant flow is also derived for an oblate spheroidal cavity of arbitrary eccentricity. Three-dimensional direct numerical simulation in an oblate spheroidal cavity is performed to demonstrate that, in both the non-resonant and resonant cases, a satisfactory agreement is achieved between the asymptotic solution and numerical simulation at $E \ll 1$.

Key words: geophysical and geological flows, rotating flows

\section{Introduction and formulation}

As a consequence of their rapid rotation as well as interaction among the Sun, planets and satellites, many celestial bodies are usually in the shape of a spheroid 
or an ellipsoid and rotating non-uniformly, resulting in libration of those bodies (Dermott 1979; William et al. 2001; Noir et al. 2009; Baland \& Van Hoolst 2010). Planetary libration represents an important dynamic characteristic that has recently been employed to examine the physical properties of planetary interiors (see e.g. Margot et al. 2007; Dufey et al. 2009; Rambaux, Van Hoolst \& Karatekin 2011).

There are usually two components of planetary libration: longitudinal (e.g. Margot et al. 2007) and latitudinal (e.g. Dufey et al. 2009). While longitudinal libration describes a periodic variation of the rotation speed of a planet around its figure (or symmetry) axis, latitudinal libration is characterized by a periodic variation of the figure axis towards and away from its mean direction. In the case of longitudinal libration, the total instantaneous angular velocity $\boldsymbol{\Omega}$ can be written as $\boldsymbol{\Omega}=\boldsymbol{\Omega}_{0}+\boldsymbol{\Omega}_{\text {long }}$, where $\Omega_{0}$ and $\boldsymbol{\Omega}_{\text {long }}$ denote the mean angular velocity and the longitudinal libration vector, respectively, $\boldsymbol{\Omega}_{0} \times \boldsymbol{\Omega}_{\text {long }}=0$ and the amplitude $\left|\boldsymbol{\Omega}_{\text {long }}\right|$ is a periodic function of time. In the case of latitudinal libration, we have $\Omega=\Omega_{0}+\Omega_{\text {lati }}$, where the fast rotation vector $\boldsymbol{\Omega}_{0}$ is fixed in an inertial frame, the latitudinal libration vector $\boldsymbol{\Omega}_{\text {lati }}$ satisfies $\boldsymbol{\Omega}_{0} \cdot \boldsymbol{\Omega}_{\text {lati }}=0$ and the amplitude $\left|\boldsymbol{\Omega}_{\text {lati }}\right|$ is a periodic function of time. Caused by similar mechanisms, the amplitude of forced longitudinal libration is likely to be of the same order as that of forced latitudinal libration. It should be pointed out, however, that planetary libration is fundamentally different from its precession, for which the total angular velocity is of the form $\boldsymbol{\Omega}=\Omega_{0}+\Omega_{\text {prec }}$, where $\Omega_{0}$ denotes the fast rotation vector around the figure axis, the precession vector $\boldsymbol{\Omega}_{\text {prec }}$ satisfies $\boldsymbol{\Omega}_{0} \times \boldsymbol{\Omega}_{\text {prec }} \neq 0$ and, more significantly, its amplitude $\left|\boldsymbol{\Omega}_{\text {prec }}\right|$ is constant. It follows that precession describes a change only in the orientation of the rotation axis of a planet. The present study is concerned with the dynamic response of a homogeneous incompressible fluid with kinematic viscosity $v$ confined within a spheroidal cavity of arbitrary eccentricity $\mathscr{E}$ to latitudinal libration. Note that spheroidal or ellipsoidal geometry has frequently been employed by many authors to study fluid motion in geophysical and astrophysical contexts (see e.g. Vanyo et al. 1995; Lorenzani \& Tilgner 2001; Noir et al. 2003; Wu \& Roberts 2009; Le Bars et al. 2010).

Three key parameters characterize the problem of librationally driven flow in spheroidal or ellipsoidal cavities: the Ekman number $E=v /\left(a^{2} \Omega_{0}\right)$, where $\Omega_{0}=\left|\Omega_{0}\right|$ is the mean rate of rotation and $a$ is the equatorial radius of the container, provides a measure of the relative importance between the typical viscous force and the Coriolis force; the eccentricity $\mathscr{E}$ measures the degree of topographic coupling between the container and its interior fluid; and the Poincaré number $P o=\left|\Omega_{\text {lati }}\right|_{\max } / \Omega_{0}$, where $\left|\boldsymbol{\Omega}_{\text {lati }}\right|_{\max }$ denotes the maximum of $\left|\boldsymbol{\Omega}_{\text {lati }}\right|$, quantifies the strength of Poincaré forcing. All three parameters are believed to be small for a typical planet: typical values of the Ekman number $E$ for many planets and satellites are extremely small, with $E \leqslant O\left(10^{-14}\right)$; the Poincaré number $P o$ is typically $O\left(10^{-4}\right)$ (see e.g. Noir et al. 2009 , table 2); and the size of the eccentricity $\mathscr{E}$ is moderately small, typically with $\mathscr{E}=O\left(10^{-1}\right)$ (Kong, Zhang \& Schubert 2010). In other words, many planets and satellites are typically marked by $E \ll 1$ and $P o \ll 1$ but with $P_{o} / E^{1 / 2} \gg 1$.

The problem of fluid motion in spherical geometry driven by longitudinal libration has been extensively studied for several decades. Aldridge \& Toomre (1969) experimentally investigated librationally driven flows in a spherical container, revealing that several axisymmetric inertial modes can be excited when the librating frequency is close to that of an inertial mode. Rieutord (1991) and Tilgner (1999) studied numerically the response to longitudinal libration in spherical systems (see also Calkins et al. 2010). The recent experimental and numerical study of Noir et al. (2009) focuses on the nonlinear property of the spherical librating flow when the Poincaré 
number $P o$ is sufficiently large. Busse (2010) showed that longitudinal libration can drive a weak mean zonal flow in librating spheres, which is confirmed by a recent experimental and numerical study (Sauret et al. 2010). In spherical geometry, coupling between the librating container and its interior fluid is purely viscous and the fluid motion driven by longitudinal libration is weak and has the typical amplitude $O(P o)$. Motivated by searching for a possible resonance with longitudinal libration, Zhang, Chan \& Liao (2011) (see also Chan, Liao \& Zhang 2011) investigated, via both analytical and numerical methods, the motion of a homogeneous fluid confined in a librating ellipsoidal cavity with moderate equatorial eccentricity $\mathscr{E}$. However, a negative conclusion is reached by both the asymptotic analysis and an extensive numerical simulation. Even when both the libration frequency and the azimuthal wavenumber of topographic forcing are exactly the same as those of non-axisymmetric inertial modes, longitudinal libration cannot resonate with any inertial modes in ellipsoidal geometry: the typical amplitude of the non-axisymmetric librating flow is $O\left(\mathscr{E}^{2} P o\right)$ for $E \ll 1$. This implies that fluid motion driven by longitudinal libration, in either spherical or ellipsoidal geometry, may not, because of small Poincaré number Po for planets, be physically significant.

The present study continues our search for a possible resonance and attempts to answer the following question. Would the dynamic response to latitudinal libration be fundamentally different from that of longitudinal libration? We consider an oblate spheroidal cavity of arbitrary eccentricity $\mathscr{E}$ described by

$$
\frac{x^{2}}{a^{2}}+\frac{y^{2}}{a^{2}}+\frac{z^{2}}{a^{2}\left(1-\mathscr{E}^{2}\right)}=1,
$$

where $0<\mathscr{E}<1$ and the $z$-axis represents the symmetry axis. The spheroidal container rotates rapidly with a mean angular velocity $\Omega_{0}$ and a small Ekman number $E \ll 1$ and undergoes weak latitudinal libration with a small Poincaré number $P o \ll 1$ and libration frequency $\Omega_{0} \hat{\omega}$, where $\hat{\omega}$ is the non-dimensional frequency. We shall unveil that, via both asymptotic and numerical analysis, latitudinal libration in spheroidal geometry can directly resonate with a spheroidal inertial mode representing an azimuthally travelling wave when the libration frequency $\hat{\omega}$ is close to $2 /\left(2-\mathscr{E}^{2}\right)$. At the resonance, the viscous boundary layer plays an essential role in determining the flow amplitude that becomes $O\left(P o / E^{1 / 2}\right)$ for $E \ll 1$. We shall derive, using oblate spheroidal coordinates and without making any prior assumptions about the spatial-temporal structure of the flow, two asymptotic solutions for the resonant and non-resonant flow satisfying the no-slip boundary condition in an oblate spheroidal cavity of arbitrary eccentricity. We shall also carry out three-dimensional direct numerical simulation, valid for both moderately large or small $E$, of the same problem for the purpose of validating our asymptotic solutions valid only for $E \ll 1$.

In what follows we shall begin by presenting the mathematical formulation of the problem in $\$ 2$. An asymptotic analysis for both non-resonant and resonant flow is discussed in $\S 3$, while fully three-dimensional numerical simulation is presented in $\S 4$. A summary and some remarks are given in $\S 5$.

\section{Mathematical formulation of the problem}

Consider a homogeneous fluid of viscosity $v$ confined within an oblate spheroidal cavity defined by (1.1). Suppose that the spheroidal container rotates rapidly with a mean angular velocity $\Omega_{0}$, which is fixed in the inertial frame and, at the same time, undergoes latitudinal libration that is marked by the frequency $\hat{\omega} \Omega_{0}$ and the amplitude 
$\left|\Omega_{\text {lati }}\right|=P o \Omega_{0}\left|\sin \left(\hat{\omega} \Omega_{0} t\right)\right|$, which results in a periodic variation of the figure axis slightly towards and away from the rotation axis $\boldsymbol{\Omega}_{0}$. Motivated by its application to synchronous planets and moons, we assume that the total angular velocity $\Omega$ of the spheroidal container can be written as

$$
\boldsymbol{\Omega}=\boldsymbol{\Omega}_{0}+\hat{\boldsymbol{x}} \Omega_{0} \operatorname{Pos} \sin \left(\hat{\omega} \Omega_{0} t\right),
$$

where $\hat{\boldsymbol{x}}$ is a unit vector that is fixed in a frame of reference attached to the container, the mantle frame of reference, and perpendicular to the mean angular velocity $\boldsymbol{\Omega}_{0}$, and $P o / \hat{\omega}$ represents the maximum angular displacement of latitudinal libration. While the mean angular velocity $\Omega_{0}$ is constant in the inertial frame, it is time-dependent in the mantle frame of reference. The total angular velocity $\Omega$ in the mantle frame can be written as

$$
\boldsymbol{\Omega}=\Omega_{0}\left\{\hat{z} \cos \left[(P o / \hat{\omega}) \cos \left(\hat{\omega} \Omega_{0} t\right)\right]-\hat{\boldsymbol{y}} \sin \left[(P o / \hat{\omega}) \cos \left(\hat{\omega} \Omega_{0} t\right)\right]+\hat{\boldsymbol{x}} P o \sin \left(\hat{\omega} \Omega_{0} t\right)\right\},
$$

where $\hat{z}$ is in the direction of the figure axis described by (1.1). This study, both theoretical and numerical, is only concerned with the problem of weakly latitudinal libration by assuming that $P o \ll 1$ and $(P o / \hat{\omega}) \ll 1$. It is worth noting that the problem of synchronous latitudinal libration, defined by (2.1), is readily realizable in laboratory experiments.

In the mantle frame of reference, the dynamics of latitudinally librational driven flow, under the assumptions $P o \ll 1$ and $(P o / \hat{\omega}) \ll 1$, is governed by the dimensional equations:

$$
\begin{gathered}
\frac{\partial \boldsymbol{u}}{\partial t}+\boldsymbol{u} \cdot \boldsymbol{\nabla} \boldsymbol{u}+2 \Omega_{0}\left[\hat{\boldsymbol{z}}+\hat{\boldsymbol{x}} P o \sin \left(\Omega_{0} \hat{\omega}\right) t-\hat{\boldsymbol{y}}(P o / \hat{\omega}) \cos \left(\Omega_{0} \hat{\omega} t\right)\right] \times \boldsymbol{u}+\frac{1}{\rho} \nabla p \\
=v \nabla^{2} \boldsymbol{u}+P o \Omega_{0}^{2}\left[\hat{\omega} \boldsymbol{r} \times \hat{\boldsymbol{x}} \cos \left(\Omega_{0} \hat{\omega} t\right)+\boldsymbol{r} \times(\hat{\boldsymbol{z}} \times \hat{\boldsymbol{x}}) \sin \left(\Omega_{0} \hat{\omega} t\right)\right], \\
\boldsymbol{\nabla} \cdot \boldsymbol{u}=0,
\end{gathered}
$$

where $\boldsymbol{r}$ is the position vector, $p$ is a reduced pressure and $\boldsymbol{u}$ is the three-dimensional velocity field. The final two terms on the right-hand side of (2.3) are known as the Poincaré force, which results from latitudinal libration and drives fluid motion in the spheroidal cavity.

Employing the semi-axis $a$ as the length scale, $\Omega_{0}^{-1}$ as the unit of time and $\rho a^{2} \Omega_{0}^{2}$ as the unit of pressure, the non-dimensional envelope of the spheroidal cavity is then described by

$$
\frac{x^{2}}{1}+\frac{y^{2}}{1}+\frac{z^{2}}{\left(1-\mathscr{E}^{2}\right)}=1
$$

while the non-dimensional governing equations are:

$$
\begin{aligned}
& \frac{\partial \boldsymbol{u}}{\partial t}+\boldsymbol{u} \cdot \nabla \boldsymbol{u}+2 \hat{z} \times \boldsymbol{u}+\nabla p= E \nabla^{2} \boldsymbol{u}+2 P o[(1 / \hat{\omega}) \hat{\boldsymbol{y}} \times \boldsymbol{u} \cos (\hat{\omega} t)-\hat{\boldsymbol{x}} \times \boldsymbol{u} \sin (\hat{\omega} t)] \\
&+ P o[\hat{\omega} \boldsymbol{r} \times \hat{\boldsymbol{x}} \cos (\hat{\omega} t)+\boldsymbol{r} \times(\hat{z} \times \hat{\boldsymbol{x}}) \sin (\hat{\omega} t)], \\
& \boldsymbol{\nabla} \cdot \boldsymbol{u}=0 .
\end{aligned}
$$

Note that the centrifugal force is combined with all other conservative forces to form the reduced pressure $p$. Librationally driven flow on the bounding surface, $\mathscr{S}$, of the oblate spheroidal cavity (2.5) is at rest, requiring that

$$
\hat{\boldsymbol{n}} \cdot \boldsymbol{u}=0
$$


and

$$
\hat{\boldsymbol{n}} \times \boldsymbol{u}=\mathbf{0},
$$

where $\hat{\boldsymbol{n}}$ denotes the normal to the bounding surface, $\mathscr{S}$, of the spheroidal cavity.

For deriving an asymptotic solution of the viscous boundary layer on $\mathscr{S}$, it is mathematically convenient to introduce oblate spheroidal coordinates $(\eta, \phi, \tau)$ with the corresponding unit vector $(\hat{\boldsymbol{\eta}}, \hat{\boldsymbol{\phi}}, \hat{\boldsymbol{\tau}})$ defined by

$$
\begin{gathered}
x^{2}=\left(\mathscr{E}^{2}+\eta^{2}\right)\left(1-\tau^{2}\right) \cos ^{2} \phi, \\
y^{2}=\left(\mathscr{E}^{2}+\eta^{2}\right)\left(1-\tau^{2}\right) \sin ^{2} \phi, \\
z^{2}=\eta^{2} \tau^{2} .
\end{gathered}
$$

In oblate spheroidal coordinates, the surfaces of constant $\eta$ form oblate spheroids, providing a set of coordinate surfaces, with the foci of all the oblate spheroids being located at $\sqrt{x^{2}+y^{2}}=\mathscr{E}$ and $z=0$; while the surfaces of constant $\tau$ form hyperboloids, offering another set of coordinate surfaces, with the foci of all the hyperboloids also being located at $\sqrt{x^{2}+y^{2}}=\mathscr{E}$ and $z=0$. The envelope of the spheroidal cavity $\mathscr{S}$ is simply given by $\eta=\sqrt{1-\mathscr{E}^{2}}$. Various differential operators can be readily cast into oblate spheroidal coordinates: for instance, the pressure gradient can be written as

$$
\begin{aligned}
\nabla p= & \hat{\boldsymbol{\eta}}\left(\frac{\sqrt{\eta^{2}+\mathscr{E}^{2}}}{\sqrt{\eta^{2}+\mathscr{E}^{2} \tau^{2}}}\right) \frac{\partial p}{\partial \eta}+\hat{\boldsymbol{\tau}}\left(\frac{\sqrt{1-\tau^{2}}}{\sqrt{\eta^{2}+\mathscr{E}^{2} \tau^{2}}}\right) \frac{\partial p}{\partial \tau} \\
& +\hat{\boldsymbol{\phi}}\left[\frac{1}{\sqrt{\left(\eta^{2}+\mathscr{E}^{2}\right)\left(1-\tau^{2}\right)}}\right] \frac{\partial p}{\partial \phi} .
\end{aligned}
$$

We shall present our results in terms of oblate spheroidal coordinates, the natural coordinates for a viscous flow within a spheroidal cavity of arbitrary eccentricity $\mathscr{E}$.

The problem defined by (2.6) and (2.7) subject to the boundary conditions (2.8) and (2.9) for spheroidal geometry (2.5) will first be solved by an asymptotic method at $E \ll 1$ in $\S 3$, and then by direct three-dimensional simulation using a finite element method in $\S 4$.

\section{Asymptotic analysis}

\subsection{Asymptotic expansion and solvability condition}

For weakly librating flow in a spheroidal cavity of arbitrary eccentricity marked by $P o \ll 1$, the nonlinear term, as well as small perturbations to the primary Coriolis force $2 \hat{z} \times \boldsymbol{u}$, may be neglected in the first approximation and the fluid motion is then governed by

$$
\begin{gathered}
\frac{\partial \boldsymbol{u}}{\partial t}+2 \hat{\boldsymbol{z}} \times \boldsymbol{u}+\nabla p=E \nabla^{2} \boldsymbol{u}+P o[\hat{\omega} \boldsymbol{r} \times \hat{\boldsymbol{x}} \cos (\hat{\omega} t)+\boldsymbol{r} \times(\hat{\boldsymbol{z}} \times \hat{\boldsymbol{x}}) \sin (\hat{\omega} t)], \\
\nabla \cdot \boldsymbol{u}=0 .
\end{gathered}
$$

It will be discussed later, however, that our asymptotic solution actually satisfies the nonlinear equation including the term $\boldsymbol{u} \cdot \nabla \boldsymbol{u}$ if the nonlinear effect within the viscous boundary layer is neglected. An asymptotic solution at $E \ll 1$ to (3.1) and (3.2) satisfying the no-slip boundary condition can be sought by assuming that inertial modes in a spheroidal cavity, which automatically satisfy (3.2) and (2.8), form a 
complete function system and thus can be employed to represent an arbitrary profile of the interior librating flow. In other words, (3.1) and (3.2) may be solved by making the following expansions at $E \ll 1$ :

$$
\begin{gathered}
\boldsymbol{u}=\sum_{n k}\left(A_{n k} \boldsymbol{u}_{n k}+\text { c.c. }+\widehat{\boldsymbol{u}}_{s}+\widetilde{\boldsymbol{u}}_{s}\right) \sin \hat{\omega} t+\sum_{n k}\left(B_{n k} \boldsymbol{u}_{n k}+\text { c.c. }+\widehat{\boldsymbol{u}}_{c}+\widetilde{\boldsymbol{u}}_{c}\right) \cos \hat{\omega} t \\
p=\sum_{n k}\left(A_{n k} p_{n k}+\text { c.c. }+\widehat{p}_{s}+\widetilde{p}_{s}\right) \sin \hat{\omega} t+\sum_{n k}\left(B_{n k} p_{n k}+\text { c.c. }+\widehat{p}_{c}+\widetilde{p}_{c}\right) \cos \hat{\omega} t
\end{gathered}
$$

where $A_{n k}$ and $B_{n k}$ are complex coefficients to be determined, c.c. denotes the complex conjugate of the preceding term, and $\boldsymbol{u}_{n k}$ and $p_{n k}$ satisfy the equations

$$
\begin{gathered}
0=\mathrm{i} 2 \sigma_{n k} \boldsymbol{u}_{n k}+2 \hat{z} \times \boldsymbol{u}_{n k}+\nabla p_{n k}, \\
0=\nabla \cdot \boldsymbol{u}_{n k},
\end{gathered}
$$

where $\mathrm{i}=\sqrt{-1}$, and $\sigma_{n k}$ is the half-frequency of a spheroidal inertial mode with $\left|\sigma_{n k}\right|<1$, subject to the boundary condition $\hat{\boldsymbol{n}} \cdot \boldsymbol{u}_{n k}=0$ at the bounding surface of a spheroid $\eta=\sqrt{1-\mathscr{E}^{2}}$. In the asymptotic expansion (3.3), the velocity $\boldsymbol{u}$ is decomposed into a viscous boundary-layer flow, $\widetilde{\boldsymbol{u}}_{s} \sin \hat{\omega} t$ and $\widetilde{\boldsymbol{u}}_{c} \cos \hat{\omega} t$, and the interior flow, which is represented by spheroidal inertial modes $\boldsymbol{u}_{n k}$. Viscous action on the inertial modes $\boldsymbol{u}_{n k}$ induces a thin viscous boundary layer on $\mathscr{S}$. By producing a normal mass flux from, or sucking the interior fluid into, the thin viscous boundary layer, the viscous effect drives the secondary interior flow $\widehat{\boldsymbol{u}}_{s} \sin \hat{\omega} t$ and $\widehat{\boldsymbol{u}}_{c} \cos \hat{\omega} t$, communicating with the interior fluid. It should be pointed out that the asymptotic expansion (3.3), without making any prior assumption, may be used to represent any physically acceptable flow for $E \ll 1$ in spheroidal geometry.

Since the Poincare forcing on the right-hand side of (3.1) is marked by the azimuthal wavenumber $m=1$, the $p_{n k}$ and $\boldsymbol{u}_{n k}$ in the asymptotic expansions (3.3) and (3.4) with the required spatial symmetry can be extracted from the general solution (Zhang, Liao \& Earnshaw 2004) and are given by

$$
\begin{aligned}
p_{n k}(\eta, \tau, \phi)= & \sum_{i=0}^{k} \sum_{j=0}^{k-i} C_{k i j} \sigma_{n k}^{2 i}\left(1-\sigma_{n k}^{2}\right)^{j} \\
& \times\left[\left(1-\tau^{2}\right)\left(\eta^{2}+\mathscr{E}^{2}\right)\right]^{(1 / 2+j)}(\eta \tau)^{2 i+1} \mathrm{e}^{\mathrm{i} \phi}, \\
\hat{\boldsymbol{\eta}} \cdot \boldsymbol{u}_{n k}(\eta, \tau, \phi)= & \mathrm{i} \sum_{i=0}^{k} \sum_{j=0}^{k-i} \frac{C_{k i j}}{2 \sqrt{\left(\eta^{2}+\mathscr{E}^{2} \tau^{2}\right)\left(\eta^{2}+\mathscr{E}^{2}\right)}} \sigma_{n k}^{2 i-1}\left(1-\sigma_{n k}^{2}\right)^{j-1} \\
& \times\left[-\eta^{2} \sigma_{n k}\left(2 j \sigma_{n k}+\sigma_{n k}+1\right)+(2 i+1)\left(\eta^{2}+\mathscr{E}^{2}\right)\left(1-\sigma_{n k}^{2}\right)\right] \\
& \times\left[\left(1-\tau^{2}\right)\left(\eta^{2}+\mathscr{E}^{2}\right)\right]^{(1 / 2+j)} \eta^{2 i} \tau^{2 i+1} \mathrm{e}^{\mathrm{i} \phi}, \\
\hat{\boldsymbol{\phi}} \cdot \boldsymbol{u}_{n k}(\eta, \tau, \phi)= & \sum_{i=0}^{k} \sum_{j=0}^{k-i} \frac{C_{k i j}}{2 \sqrt{\left(1-\tau^{2}\right)\left(\eta^{2}+\mathscr{E}^{2}\right)}} \sigma_{n k}^{2 i}\left(1-\sigma_{n k}^{2}\right)^{j-1} \\
& \times\left(2 j+1+\sigma_{n k}\right)\left[\left(1-\tau^{2}\right)\left(\eta^{2}+\mathscr{E}^{2}\right)\right]^{(1 / 2+j)}(\eta \tau)^{2 i+1} \mathrm{e}^{\mathrm{i} \phi},
\end{aligned}
$$




$$
\begin{aligned}
\hat{\boldsymbol{\tau}} \cdot \boldsymbol{u}_{n k}(\eta, \tau, \phi)= & \mathrm{i} \sum_{i=0}^{k} \sum_{j=0}^{k-i} \frac{C_{k i j}}{2 \sqrt{\left(1-\tau^{2}\right)\left(\eta^{2}+\mathscr{E}^{2} \tau^{2}\right)}} \sigma_{n k}^{2 i-1}\left(1-\sigma_{n k}^{2}\right)^{j-1} \\
& \times\left[\tau^{2} \sigma_{n k}\left(2 j \sigma_{n k}+\sigma_{n k}+1\right)+(2 i+1)\left(1-\tau^{2}\right)\left(1-\sigma_{n k}^{2}\right)\right] \\
& \times\left[\left(1-\tau^{2}\right)\left(\eta^{2}+\mathscr{E}^{2}\right)\right]^{(1 / 2+j)} \eta^{2 i+1} \tau^{2 i} \mathrm{e}^{\mathrm{i} \phi},
\end{aligned}
$$

with an arbitrary normalization for mathematical convenience. Here $k \geqslant 0, n$ is restricted by $1 \leqslant n \leqslant(2 k+1)$, the indices $(n, k)$ are roughly related to the spatial wavenumbers in the vertical and radial directions respectively, and $C_{k i j}$ is

$$
C_{k i j}=\left[\frac{-1}{\left(1-\sigma_{n k}^{2} \mathscr{E}^{2}\right)}\right]^{i+j} \frac{[2(k+i+j)+3] ! !}{2^{j+1}(2 i+1) ! !(k-i-j) ! i j ! !(1+j) !},
$$

along with $(2 i-1) ! !=(2 i-1) \cdots(3)(1),(-1) ! !=1$ and $(0) !=1$. The half-frequencies of the spheroidal inertial modes in (3.7)-(3.10), $\sigma_{n k}$, are solutions of

$$
\begin{aligned}
0= & \sum_{j=0}^{k}(-1)^{j} \frac{[2(2 k+2-j)] !}{[2(k-j)+1] ! j !(2 k+2-j) !} \\
& \times\left[1-\frac{\left(1-\sigma_{n k}\right)[2(k-j)+1]}{\sigma_{n k}\left(1-\mathscr{E}^{2}\right)}\right]\left[\frac{\left(1-\mathscr{E}^{2}\right) \sigma_{n k}^{2}}{\left(1-\sigma_{n k}^{2} \mathscr{E}^{2}\right)}\right]^{k-j} \quad \text { with } k \geqslant 0 .
\end{aligned}
$$

For any given $\mathscr{E}$ and $k$, there exist $(2 k+1)$ different real solutions for (3.12) corresponding to the $(2 k+1)$ different inertial modes, which can be arranged according to the size of $\left|\sigma_{n k}\right|$,

$$
0<\left|\sigma_{1 k}\right|<\left|\sigma_{2 k}\right|<\left|\sigma_{3 k}\right|<\cdots<\left|\sigma_{n k}\right|<\cdots .
$$

In other words, the index $n$ for $\sigma_{n k}$ in (3.7)-(3.10) denotes the $n$th smallest root of (3.12) for given $k$.

Of the spheroidal inertial modes represented by (3.7)-(3.10), the simplest is obviously given by setting $k=0$, which corresponds to a single inertial mode with $n=1$. The details of this spheroidal inertial mode, $\boldsymbol{u}_{10}$ and $p_{10}$, can be easily derived from the general expressions (3.7)-(3.10). While the solution of (3.12) at $k=0$ gives

$$
\sigma_{10}=\frac{1}{2-\mathscr{E}^{2}},
$$

the expressions (3.7)-(3.10) at $k=0$, together with (3.14), yield

$$
\begin{gathered}
p_{10}(\eta, \tau, \phi)=\frac{3}{2}\left(\eta^{2}+\mathscr{E}^{2}\right)^{1 / 2}\left(1-\tau^{2}\right)^{1 / 2} \eta \tau \mathrm{e}^{\mathrm{i} \phi}, \\
\hat{\boldsymbol{\eta}} \cdot \boldsymbol{u}_{10}(\eta, \tau, \phi)=\mathrm{i}\left[\frac{3\left(2-\mathscr{E}^{2}\right)}{4\left(1-\mathscr{E}^{2}\right)}\right] \frac{\tau \mathscr{E}^{2} \sqrt{1-\tau^{2}}\left(1-\mathscr{E}^{2}-\eta^{2}\right)}{\sqrt{\eta^{2}+\tau^{2} \mathscr{E}^{2}}} \mathrm{e}^{\mathrm{i} \phi}, \\
\hat{\boldsymbol{\phi}} \cdot \boldsymbol{u}_{10}(\eta, \tau, \phi)=\left[\frac{3\left(2-\mathscr{E}^{2}\right)}{4\left(1-\mathscr{E}^{2}\right)}\right] \eta \tau \mathrm{e}^{\mathrm{i} \phi}, \\
\hat{\boldsymbol{\tau}} \cdot \boldsymbol{u}_{10}(\eta, \tau, \phi)=\mathrm{i}\left[\frac{3\left(2-\mathscr{E}^{2}\right)}{4\left(1-\mathscr{E}^{2}\right)}\right] \frac{\eta \sqrt{\eta^{2}+\mathscr{E}^{2}}\left(1-\mathscr{E}^{2}+\tau^{2} \mathscr{E}^{2}\right)}{\sqrt{\eta^{2}+\tau^{2} \mathscr{E}^{2}}} \mathrm{e}^{\mathrm{i} \phi} .
\end{gathered}
$$

This non-axisymmetric inertial mode is, in some ways, analogous to the spin-over mode (see e.g. Greenspan 1968) but represents an azimuthally, retrogradely travelling 
wave with the phase speed $2 /\left(2-\mathscr{E}^{2}\right)$. Furthermore, $\boldsymbol{u}_{10} \mathrm{e}^{\mathrm{i} 2 t /\left(2-\mathscr{E}^{2}\right)}$ and $p_{10} \mathrm{e}^{\mathrm{i} 2 t /\left(2-\mathscr{E}^{2}\right)}$ are an exact solution in closed form to

$$
\begin{gathered}
\frac{\partial\left[\boldsymbol{u}_{10} \mathrm{e}^{\mathrm{i} 2 t /\left(2-\mathscr{E}^{2}\right)}\right]}{\partial t}+2 \hat{z} \times\left[\boldsymbol{u}_{10} \mathrm{e}^{\mathrm{i} 2 t /\left(2-\mathscr{E}^{2}\right)}\right]+\nabla\left[p_{10} \mathrm{e}^{\mathrm{i} 2 t /\left(2-\mathscr{E}^{2}\right)}\right]=E \nabla^{2}\left[\boldsymbol{u}_{10} \mathrm{e}^{\mathrm{i} 2 t /\left(2-\mathscr{E}^{2}\right)}\right], \\
\nabla \cdot\left[\boldsymbol{u}_{10} \mathrm{e}^{\mathrm{i} 2 t /\left(2-\mathscr{E}^{2}\right)}\right]=0,
\end{gathered}
$$

in a spheroidal cavity of arbitrary eccentricity $0 \leqslant \mathscr{E}<1$ satisfying $\hat{\boldsymbol{n}} \cdot \boldsymbol{u}_{10}=0$ at the bounding surface of the cavity $\eta=\sqrt{1-\mathscr{E}^{2}}$.

A major task of our asymptotic analysis is then to find the analytical expression for coefficients $A_{n k}$ and $B_{n k}$, as well as the viscous boundary layer $\widetilde{\boldsymbol{u}}_{c}$ and $\widetilde{\boldsymbol{u}}_{s}$, in the expansions (3.3) and (3.4). After substituting the expansions into (3.1), multiplying the resulting equation by $\boldsymbol{u}_{n k}^{*}$, the complex conjugate of $\boldsymbol{u}_{n k}$, and then integrating over the spheroid, we obtain two solvability conditions:

$$
\begin{aligned}
& A_{n k}\left(\hat{\omega}^{2}-\omega_{n k}^{2}\right) \int_{V}\left|\boldsymbol{u}_{n k}\right|^{2} \mathrm{~d} V+\hat{\omega} \int_{V} \boldsymbol{u}_{n k}^{*} \cdot\left(\hat{\omega} \widehat{\boldsymbol{u}}_{s}+2 \hat{z} \times \widehat{\boldsymbol{u}}_{c}+\nabla \widehat{p}_{c}\right) \mathrm{d} V \\
& -\mathrm{i} \omega_{n k} \int_{V} \boldsymbol{u}_{n k}^{*} \cdot\left(-\hat{\omega} \widehat{\boldsymbol{u}}_{c}+2 \hat{z} \times \widehat{\boldsymbol{u}}_{s}+\nabla \widehat{p}_{s}\right) \mathrm{d} V \\
& \quad=P o \int_{V} \boldsymbol{u}_{n k}^{*} \cdot\left[\hat{\omega}^{2} \boldsymbol{r} \times \hat{\boldsymbol{x}}-\mathrm{i} \omega_{n k} \boldsymbol{r} \times(\hat{z} \times \hat{\boldsymbol{x}})\right] \mathrm{d} V, \\
& \mathrm{i} B_{n k}\left(\hat{\omega}^{2}-\omega_{n k}^{2}\right) \int_{V}\left|\boldsymbol{u}_{n k}\right|^{2} \mathrm{~d} V+\omega_{n k} \int_{V} \boldsymbol{u}_{n k}^{*} \cdot\left(\hat{\omega} \widehat{\boldsymbol{u}}_{s}+2 \hat{z} \times \widehat{\boldsymbol{u}}_{c}+\nabla \widehat{p}_{c}\right) \mathrm{d} V \\
& -\mathrm{i} \hat{\omega} \int_{V} \boldsymbol{u}_{n k}^{*} \cdot\left(-\hat{\omega} \widehat{\boldsymbol{u}}_{c}+2 \hat{z} \times \widehat{\boldsymbol{u}}_{s}+\nabla \widehat{p}_{s}\right) \mathrm{d} V \\
& \quad=\hat{\omega} P o \int_{V} \boldsymbol{u}_{n k}^{*} \cdot\left[\omega_{n k} \boldsymbol{r} \times \hat{\boldsymbol{x}}-\mathrm{i} \boldsymbol{r} \times(\hat{z} \times \hat{\boldsymbol{x}})\right] \mathrm{d} V,
\end{aligned}
$$

where $\omega_{n k}=2 \sigma_{n k}, k \geqslant 0,1 \leqslant n \leqslant(2 k+1)$ and $\int_{V}$ denotes the volume integration over the spheroidal cavity. Meanwhile, there are also two equations for the viscous boundary layer:

$$
\begin{gathered}
\hat{\omega} \widetilde{\boldsymbol{u}}_{s}+2 \hat{z} \times \widetilde{\boldsymbol{u}}_{c}+\hat{\boldsymbol{n}}\left(\hat{\boldsymbol{n}} \cdot \nabla \widetilde{p}_{c}\right)=\frac{\partial^{2} \widetilde{\boldsymbol{u}}_{c}}{\partial \xi^{2}}, \\
-\hat{\omega} \widetilde{\boldsymbol{u}}_{c}+2 \hat{z} \times \widetilde{\boldsymbol{u}}_{s}+\hat{\boldsymbol{n}}\left(\hat{\boldsymbol{n}} \cdot \nabla \widetilde{p}_{s}\right)=\frac{\partial^{2} \widetilde{\boldsymbol{u}}_{s}}{\partial \xi^{2}} .
\end{gathered}
$$

Here we have introduced a stretched boundary-layer variable $\xi=E^{1 / 2}\left[\left(1-\mathscr{E}^{2}\right)^{1 / 2}-\eta\right]$ for which $\hat{\boldsymbol{n}} \cdot \nabla=-E^{-1 / 2} \partial / \partial \xi$ with $\xi=0$ at $\mathscr{S}$ while $\xi=\infty$ defines the outer edge of the viscous boundary layer. The no-slip boundary condition (2.9) implies that $\widetilde{\boldsymbol{u}}_{s}$ and $\widetilde{\boldsymbol{u}}_{c}$ are subject to the following boundary conditions on $\mathscr{S}$ :

$$
\begin{aligned}
\widetilde{\boldsymbol{u}}_{s} & =-\sum_{n k}\left[A_{n k} \boldsymbol{u}_{n k}+\text { c.c. }\right]_{\eta=\sqrt{1-\mathscr{E}^{2}}}, \\
\widetilde{\boldsymbol{u}}_{c} & =-\sum_{n k}\left[B_{n k} \boldsymbol{u}_{n k}+\text { c.c. }\right]_{\eta=\sqrt{1-\mathscr{E}^{2}}} .
\end{aligned}
$$

It will be seen that the way of solving (3.21)-(3.22) and (3.23)-(3.24) for non-resonant librating flow is quite different from that for resonant flow. For this reason, we shall discuss the two different cases separately. 


\subsection{Non-resonant flow with a passive boundary layer}

At an asymptotically small Ekman number with $0<E \ll 1$, the normal mass flux from, or sucking the interior fluid into, the viscous boundary layer is of the order $E^{1 / 2}$. When $\left|\hat{\omega}^{2}-\omega_{n k}^{2}\right| \gg O\left(E^{1 / 2}\right)$, the four integrals in the two solvability conditions (3.21) and (3.22) that are connected with the secondary flow, $\widehat{\boldsymbol{u}}_{s}$ and $\widehat{\boldsymbol{u}}_{c}$, can be neglected to leading-order approximation. After carrying out the volume integration on the right-hand sides of (3.21) and (3.22), the two solvability conditions become

$$
\begin{aligned}
& A_{n k}\left(\hat{\omega}^{2}-\omega_{n k}^{2}\right) \int_{V}\left|\boldsymbol{u}_{n k}\right|^{2} \mathrm{~d} V=\omega_{n k} P o\left[\frac{\hat{\omega}^{2}\left(1-\mathscr{E}^{2}\right)}{\left(2-\omega_{n k}\right)}-1\right] \mathscr{I}_{n k}, \\
& \mathrm{i} B_{n k}\left(\hat{\omega}^{2}-\omega_{n k}^{2}\right) \int_{V}\left|\boldsymbol{u}_{n k}\right|^{2} \mathrm{~d} V=\hat{\omega} P o\left[\frac{\omega_{n k}\left(1-\mathscr{E}^{2}\right)}{\left(2-\omega_{n k}\right)}-1\right] \mathscr{I}_{n k},
\end{aligned}
$$

where $\left|\omega_{n k}\right|<2$ and $\mathscr{I}_{n k}$ represents a two-dimensional summation for given $\sigma_{n k}$ and $\mathscr{E}$,

$$
\begin{aligned}
\mathscr{I}_{n k}= & \frac{2 \pi \sqrt{1-\mathscr{E}^{2}}}{\sigma_{n k}} \sum_{i=0}^{k} \sum_{j=0}^{k-i}(-1)^{i+j}\left[\frac{\sigma_{n k}^{2}\left(1-\mathscr{E}^{2}\right)}{\left(1-\sigma_{n k}^{2} \mathscr{E}^{2}\right)}\right]^{i}\left[\frac{\left(1-\sigma_{n k}^{2}\right)}{\left(1-\sigma_{n k}^{2} \mathscr{E}^{2}\right)}\right]^{j} \\
& \times\left[\frac{[2(k+i+j)+3] ! !}{(2 i+2 j+5) ! !(k-i-j) ! i ! j !}\right] .
\end{aligned}
$$

In order to determine $A_{n k}$ and $B_{n k}$ for all possible $n$ and $k$, it is essential to evaluate the summation $\mathscr{I}_{n k}$ given by (3.29). When $k=0$, the summation (3.29), together with $\sigma_{10}$ given by (3.14), can be readily evaluated to give

$$
\mathscr{I}_{10}=\frac{2 \pi\left(2-\mathscr{E}^{2}\right) \sqrt{1-\mathscr{E}^{2}}}{5} .
$$

When $k=1$, we can also easily make a direct summation of (3.29), which gives

$$
\begin{aligned}
\mathscr{I}_{n 1}= & \frac{2 \pi \sqrt{1-\mathscr{E}^{2}}}{\sigma_{n 1}} \sum_{i=0}^{1} \sum_{j=0}^{1-i}(-1)^{i+j} \frac{\sigma_{n 1}^{2 i}\left(1-\sigma_{n 1}^{2}\right)^{j}}{\left(1-\sigma_{n 1}^{2} \mathscr{E}^{2}\right)^{i+j}} \\
& \times\left[\frac{[2(k+i+j)+3] ! !\left(1-\mathscr{E}^{2}\right)^{i}}{(2 i+2 j+5) ! !(k-i-j) ! i ! j !}\right] \equiv 0
\end{aligned}
$$

for any value of $\sigma_{n 1}$ with $0<\left|\sigma_{n 1}\right|<1$. When $k \geqslant 2$, the two indices $(i, j)$ in the summation (3.29) are so intimately entangled that a direct summation becomes difficult. It is found, however, that the summation $\mathscr{I}_{n k}$ also vanishes identically for all $k \geqslant 2$. Since this mathematical property is central to our asymptotic analysis, we shall present a brief direct proof showing that $\mathscr{I}_{n k} \equiv 0$ for $k \geqslant 2$.

An important step in the proof is to establish a recurrence relationship that links the large- $k$ summations of (3.29) with the small- $k$ ones, like (3.31) for $k=1$, which can then be evaluated directly. For this purpose, we introduce one additional index, $M$, by considering a new summation

$$
\begin{aligned}
\mathscr{P}_{n k}^{M}= & \frac{2 \pi \sqrt{1-\mathscr{E}^{2}}}{\sigma_{n k}} \sum_{i=0}^{k-M} \sum_{j=0}^{k-i-M}(-1)^{i+j}\left[\frac{\sigma_{n k}^{2}\left(1-\mathscr{E}^{2}\right)}{\left(1-\sigma_{n k}^{2} \mathscr{E}^{2}\right)}\right]^{i}\left[\frac{\left(1-\sigma_{n k}^{2}\right)}{\left(1-\sigma_{n k}^{2} \mathscr{E}^{2}\right)}\right]^{j} \\
& \times\left[\frac{[2(k+i+j)+3] ! !}{[2(i+j+M)+5] ! !(k-i-j-M) ! i ! j !}\right]
\end{aligned}
$$


where $k \geqslant 2$ with $(k-M) \geqslant 1$. Obviously, $\mathscr{I}_{n k}=\mathscr{P}_{n k}^{0}$. At first glance, the new summation (3.32) appears to be more complicated than the original (3.29). But it can be shown or verified that $\mathscr{P}_{n k}^{M}$ obeys the following recurrence relationship:

$$
\mathscr{P}_{n k}^{M}=\left[\frac{2(M+1-k)}{k-M}\right] \mathscr{P}_{n k}^{M+1},
$$

which implies that

$$
\begin{aligned}
\mathscr{P}_{n k}^{0} & =\left[\frac{-2(k-1)}{k}\right] \mathscr{P}_{n k}^{1}=\left[\frac{(-2)^{2}(k-1)(k-2)}{k(k-1)}\right] \mathscr{P}_{n k}^{2}=\cdots \\
& =\left[\frac{(-2)^{k-1}(k-1) !}{k !}\right] \mathscr{P}_{n k}^{k-1} .
\end{aligned}
$$

In other words, we have established that

$$
\mathscr{I}_{n k}=\left[\frac{(-2)^{k-1}}{k}\right] \mathscr{P}_{n k}^{k-1} .
$$

At $M=k-1$, the summation (3.32) can be easily carried out:

$$
\begin{aligned}
\mathscr{P}_{n k}^{k-1}= & \frac{2 \pi \sqrt{1-\mathscr{E}^{2}}}{\sigma_{n k}} \sum_{i=0}^{1} \sum_{j=0}^{1-i}(-1)^{i+j}\left[\frac{\sigma_{n k}^{2}\left(1-\mathscr{E}^{2}\right)}{\left(1-\sigma_{n k}^{2} \mathscr{E}^{2}\right)}\right]^{i}\left[\frac{\left(1-\sigma_{n k}^{2}\right)}{\left(1-\sigma_{n k}^{2} \mathscr{E}^{2}\right)}\right]^{j} \\
& \times\left[\frac{1}{(1-i-j) ! i j j !}\right] \equiv 0 .
\end{aligned}
$$

By virtue of the recurrence relationship (3.35) and the summation (3.36) at $M=k-1$, we conclude that

$$
\mathscr{I}_{n k} \equiv 0 \quad \text { for } k \geqslant 2 .
$$

On the basis of (3.27), (3.28), (3.30) and (3.37), we obtain that

$$
A_{n k}=0, \quad B_{n k}=0 \quad \text { when } n \neq 1, k \neq 0
$$

and

$$
A_{10}=\frac{4 P o\left(1-\mathscr{E}^{2}\right)\left[\hat{\omega}^{2}\left(2-\mathscr{E}^{2}\right)-2\right]}{3\left[\hat{\omega}^{2}\left(2-\mathscr{E}^{2}\right)^{2}-4\right]\left(2-\mathscr{E}^{2}\right)}, \quad B_{10}=-\frac{\mathrm{i} 4 P o \hat{\omega} \mathscr{E}^{2}\left(1-\mathscr{E}^{2}\right)}{3\left[\hat{\omega}^{2}\left(2-\mathscr{E}^{2}\right)^{2}-4\right]\left(2-\mathscr{E}^{2}\right)}
$$

This implies that, regardless of the size of the libration frequency $\hat{\omega}$, only the spheroidal inertial mode $\boldsymbol{u}_{10}$ is sustained by latitudinal libration. Substitution of $A_{n k}$ and $B_{n k}$ into the expansion (3.3) gives rise to an interior solution for the librationally driven flow in a spheroidal cavity that does not satisfy the no-slip boundary condition.

A complete asymptotic solution of a viscous flow at $E \ll 1$ requires a boundarylayer flow, $\widetilde{\boldsymbol{u}}_{s}$ and $\widetilde{\boldsymbol{u}}_{c}$. It should be pointed out that, since the librating flow is generally oscillatory, with its amplitude being a function of time $t$, its boundary-layer analysis is slightly more complicated than that for a travelling wave that has constant amplitude. For an oscillatory flow, $\widetilde{\boldsymbol{u}}_{s}$ and $\widetilde{\boldsymbol{u}}_{c}$ in the expansion (3.3) have to be treated separately. Mathematically, it may be convenient to derive the two fourth-order differential equations by applying the operators $\hat{\boldsymbol{n}} \times$ and $\hat{\boldsymbol{n}} \times \hat{\boldsymbol{n}} \times$ to (3.23) and (3.24), and then combining the two resulting equations, which yield

$$
\left(\frac{\partial^{2}}{\partial \xi^{2}}-\frac{2 \mathrm{i} \tau}{\sqrt{1-\mathscr{E}^{2}+\mathscr{E}^{2} \tau^{2}}}\right)^{2}\left(\mathrm{i} \widetilde{\boldsymbol{u}}_{c}+\hat{\boldsymbol{n}} \times \widetilde{\boldsymbol{u}}_{c}\right)+\hat{\omega}^{2}\left(\mathrm{i}_{c}+\hat{\boldsymbol{n}} \times \widetilde{\boldsymbol{u}}_{c}\right)=0
$$


and

$$
\left(\frac{\partial^{2}}{\partial \xi^{2}}-\frac{2 \mathrm{i} \tau}{\sqrt{1-\mathscr{E}^{2}+\mathscr{E}^{2} \tau^{2}}}\right)^{2}\left(\mathrm{i} \widetilde{\boldsymbol{u}}_{s}+\hat{\boldsymbol{n}} \times \widetilde{\boldsymbol{u}}_{s}\right)+\hat{\omega}^{2}\left(\mathrm{i} \widetilde{\boldsymbol{u}}_{s}+\hat{\boldsymbol{n}} \times \widetilde{\boldsymbol{u}}_{s}\right)=0,
$$

subject to the following boundary conditions:

$$
\begin{gathered}
{\left[\widetilde{\boldsymbol{u}}_{c}\right]_{\xi=0}=\widetilde{\boldsymbol{V}}_{c}=-\frac{2 P o \mathscr{E}^{2} \hat{\omega} \sqrt{1-\mathscr{E}^{2}}}{\left[\hat{\omega}^{2}\left(2-\mathscr{E}^{2}\right)^{2}-4\right]}\left[\left(\cos \phi \sqrt{1-\mathscr{E}^{2}+\tau^{2} \mathscr{E}^{2}}\right) \hat{\boldsymbol{\tau}}+(\tau \sin \phi) \hat{\boldsymbol{\phi}}\right]} \\
{\left[\widetilde{\boldsymbol{u}}_{s}\right]_{\xi=0}=\widetilde{\boldsymbol{V}}_{s}=\frac{2 P o\left[\hat{\omega}^{2}\left(2-\mathscr{E}^{2}\right)-2\right] \sqrt{1-\mathscr{E}^{2}}}{\left[\hat{\omega}^{2}\left(2-\mathscr{E}^{2}\right)^{2}-4\right]}} \\
\times\left[\left(\sin \phi \sqrt{1-\mathscr{E}^{2}+\tau^{2} \mathscr{E}^{2}}\right) \hat{\boldsymbol{\tau}}-(\tau \cos \phi) \hat{\boldsymbol{\phi}}\right] \\
{\left[\frac{\partial^{2}}{\partial \xi^{2}}-\frac{2 \mathrm{i} \tau}{\sqrt{\left.1-\mathscr{E}^{2}+\mathscr{E}^{2}\right]^{2}}}\right)\left[\mathrm{i} \widetilde{\boldsymbol{u}}_{c}+\hat{\boldsymbol{n}} \times \widetilde{\boldsymbol{u}}_{c}\right]_{\xi=0}=\hat{\omega}\left(\mathrm{i} \widetilde{\boldsymbol{V}}_{s}+\hat{\boldsymbol{n}} \times \widetilde{\boldsymbol{V}}_{s}\right),} \\
\left(\frac{\partial^{2}}{\partial \xi^{2}}-\frac{2 \mathrm{i} \tau}{\sqrt{1-\mathscr{E}^{2}+\mathscr{E}^{2} \tau^{2}}}\right)\left[\tilde{\boldsymbol{u}}_{s}+\hat{\boldsymbol{n}} \times \widetilde{\boldsymbol{u}}_{s}\right]_{\xi=0}=-\hat{\omega}\left(\mathrm{i} \widetilde{\boldsymbol{V}}_{c}+\hat{\boldsymbol{n}} \times \widetilde{\boldsymbol{V}}_{c}\right), \\
\left(\frac{\partial^{2}}{\partial \xi^{2}}-\frac{2 \mathrm{i} \tau}{\sqrt{1-\mathscr{E}^{2}+\mathscr{E}^{2} \tau^{2}}}\right)\left[\mathrm{i} \widetilde{\boldsymbol{u}}_{c}+\hat{\boldsymbol{n}} \times \widetilde{\boldsymbol{u}}_{c}\right]_{\xi=\infty}=0, \\
\left(\frac{\partial^{2}}{\partial \xi^{2}}-\frac{2 \mathrm{i} \tau}{\sqrt{1-\mathscr{E}^{2}+\mathscr{E}^{2} \tau^{2}}}\right)\left[\mathrm{i} \widetilde{\boldsymbol{u}}_{s}+\hat{\boldsymbol{n}} \times \widetilde{\boldsymbol{u}}_{s}\right]_{\xi=\infty}=0 .
\end{gathered}
$$

The above boundary conditions are derived from (3.25) and (3.26) together with the given values of $A_{n k}$ and $B_{n k}$ as well as the definition of a viscous boundary layer (Greenspan 1968). This boundary-layer problem can be solved to give the solutions:

$$
\begin{aligned}
\widetilde{\boldsymbol{u}}_{c}= & \frac{P o \sqrt{1-\mathscr{E}^{2}}}{\left[\hat{\omega}^{2}\left(2-\mathscr{E}^{2}\right)^{2}-4\right]}\left\{\left[\left(\hat{\omega}^{2}\left(2-\mathscr{E}^{2}\right)-2\right)\left(\sin \phi \sqrt{1-\mathscr{E}^{2}+\mathscr{E}^{2} \tau^{2}}+\mathrm{i} \tau \cos \phi\right)\right.\right. \\
& \left.-\mathscr{E}^{2} \hat{\omega}\left(\mathrm{i} \cos \phi \sqrt{1-\mathscr{E}^{2}+\mathscr{E}^{2} \tau^{2}}+\mathrm{i} \tau \sin \phi\right)\right] \hat{\tau} \mathrm{e}^{\alpha^{+} \xi} \\
& -\left[\left(\hat{\omega}^{2}\left(2-\mathscr{E}^{2}\right)-2\right)\left(\sin \phi \sqrt{1-\mathscr{E}^{2}+\mathscr{E}^{2} \tau^{2}}+\mathrm{i} \tau \cos \phi\right)\right. \\
& \left.-\mathscr{E}^{2} \hat{\omega}\left(\mathrm{i} \cos \phi \sqrt{1-\mathscr{E}^{2}+\mathscr{E}^{2} \tau^{2}}+\mathrm{i} \tau \sin \phi\right)\right] \hat{\boldsymbol{\tau}} \mathrm{e}^{\alpha^{-} \xi} \\
& +\left[\left(\hat{\omega}^{2}\left(2-\mathscr{E}^{2}\right)-2\right)\left(\mathrm{i} \sin \phi \sqrt{1-\mathscr{E}^{2}+\mathscr{E}^{2} \tau^{2}}-\tau \cos \phi\right)\right. \\
& \left.+\mathscr{E}^{2} \hat{\omega}\left(\cos \phi \sqrt{1-\mathscr{E}^{2}+\mathscr{E}^{2} \tau^{2}}-\mathrm{i} \tau \sin \phi\right)\right] \hat{\boldsymbol{\phi}} \mathrm{e}^{\alpha^{+} \xi} \\
& +\left[\left(\hat{\omega}^{2}\left(2-\mathscr{E}^{2}\right)-2\right)\left(-\mathrm{i} \sin \phi \sqrt{1-\mathscr{E}^{2}+\mathscr{E}^{2} \tau^{2}}+\tau \cos \phi\right)\right. \\
& \left.\left.+\mathscr{E}^{2} \hat{\omega}\left(\cos \phi \sqrt{1-\mathscr{E}^{2}+\mathscr{E}^{2} \tau^{2}}-\mathrm{i} \tau \sin \phi\right)\right] \hat{\boldsymbol{\phi}} \mathrm{e}^{\alpha^{-} \xi}\right\}
\end{aligned}
$$

and

$$
\begin{aligned}
\widetilde{\boldsymbol{u}}_{s}= & \frac{P o \sqrt{1-\mathscr{E}^{2}}}{\left[\hat{\omega}^{2}\left(2-\mathscr{E}^{2}\right)^{2}-4\right]}\left\{\left[\left(\hat{\omega}^{2}\left(2-\mathscr{E}^{2}\right)-2\right)\left(\mathrm{i} \sin \phi \sqrt{1-\mathscr{E}^{2}+\mathscr{E}^{2} \tau^{2}}-\tau \cos \phi\right)\right.\right. \\
& \left.+\mathscr{E}^{2} \hat{\omega}\left(\cos \phi \sqrt{1-\mathscr{E}^{2}+\mathscr{E}^{2} \tau^{2}}-\mathrm{i} \tau \sin \phi\right)\right] \hat{\boldsymbol{\tau}} \mathrm{e}^{\alpha^{+} \xi} \\
& +\left[\left(\hat{\omega}^{2}\left(2-\mathscr{E}^{2}\right)-2\right)\left(\mathrm{i} \sin \phi \sqrt{1-\mathscr{E}^{2}+\mathscr{E}^{2} \tau^{2}}-\tau \cos \phi\right)\right. \\
& \left.+\mathscr{E}^{2} \hat{\omega}\left(-\cos \phi \sqrt{1-\mathscr{E}^{2}+\mathscr{E}^{2} \tau^{2}}+\mathrm{i} \tau \sin \phi\right)\right] \hat{\boldsymbol{\tau}} \mathrm{e}^{\alpha^{-} \xi}
\end{aligned}
$$




$$
\begin{aligned}
& -\left[\left(\hat{\omega}^{2}\left(2-\mathscr{E}^{2}\right)-2\right)\left(\sin \phi \sqrt{1-\mathscr{E}^{2}+\mathscr{E}^{2} \tau^{2}}+\mathrm{i} \tau \cos \phi\right)\right. \\
& \left.+\mathscr{E}^{2} \hat{\omega}\left(\mathrm{i} \cos \phi \sqrt{1-\mathscr{E}^{2}+\mathscr{E}^{2} \tau^{2}}+\tau \sin \phi\right)\right] \hat{\boldsymbol{\phi}} \mathrm{e}^{\alpha^{+} \xi} \\
& -\left[\left(\hat{\omega}^{2}\left(2-\mathscr{E}^{2}\right)-2\right)\left(\sin \phi \sqrt{1-\mathscr{E}^{2}+\mathscr{E}^{2} \tau^{2}}+\mathrm{i} \tau \cos \phi\right)\right. \\
& \left.\left.-\mathscr{E}^{2} \hat{\omega}\left(\mathrm{i} \cos \phi \sqrt{1-\mathscr{E}^{2}+\mathscr{E}^{2} \tau^{2}}+\tau \sin \phi\right)\right] \hat{\phi} \mathrm{e}^{\alpha^{-} \xi}\right\}
\end{aligned}
$$

where

$$
\alpha^{ \pm}=-\frac{\left(1+\mathrm{i} \mathscr{S}^{ \pm}\right)}{\sqrt{2}}\left|\frac{2 \tau}{\sqrt{1-\mathscr{E}^{2}+\mathscr{E}^{2} \tau^{2}}} \pm \hat{\omega}\right|^{1 / 2}
$$

with $\mathscr{S}^{ \pm}$denoting a sign function defined as

$$
\mathscr{S}^{ \pm}=\frac{2 \tau \pm \hat{\omega} \sqrt{1-\mathscr{E}^{2}+\mathscr{E}^{2} \tau^{2}}}{\left|2 \tau \pm \hat{\omega} \sqrt{1-\mathscr{E}^{2}+\mathscr{E}^{2} \tau^{2}}\right|}
$$

Only the imaginary parts of expressions (3.49) and (3.50) are taken as the solution for $\widetilde{\boldsymbol{u}}_{c}$ and $\widetilde{\boldsymbol{u}}_{s}$. Across the viscous boundary layer, the librating flow undergoes a smooth but rapid adjustment to the interior solution and, moreover, the boundary layer plays only a passive role such that the flow satisfies the no-slip boundary condition. There exist, however, two critical co-latitudes $\tau_{c}= \pm \hat{\omega} \sqrt{1-\mathscr{E}^{2}} / \sqrt{4-\hat{\omega}^{2} \mathscr{E}^{2}}$ at which the boundary-layer solutions break down. As confirmed by the result of our numerical simulation, those singularities seem to have insignificant influences on the librating flow, which will be discussed in more depth later.

Substitution of $A_{n k}$ and $B_{n k}$, together with $\widetilde{\boldsymbol{u}}_{c}$ and $\widetilde{\boldsymbol{u}}_{s}$, into the asymptotic expansion (3.3) yields the leading-order solution of the fluid motion satisfying the no-slip boundary condition driven by latitudinal libration,

$$
\boldsymbol{u}=\left[\frac{2 P o\left(\hat{\omega}^{2}\left(2-\mathscr{E}^{2}\right)^{2}-2\right) \boldsymbol{Q}_{s}}{\left[\hat{\omega}^{2}\left(2-\mathscr{E}^{2}\right)^{2}-4\right]}+\widetilde{\boldsymbol{u}}_{s}\right] \sin \hat{\omega} t+\left[\frac{2 P o \mathscr{E}^{2} \hat{\omega} \boldsymbol{Q}_{c}}{\left[\hat{\omega}^{2}\left(2-\mathscr{E}^{2}\right)^{2}-4\right]}+\widetilde{\boldsymbol{u}}_{c}\right] \cos \hat{\omega} t
$$

where $\boldsymbol{Q}_{c}$ and $\boldsymbol{Q}_{s}$ are associated with the real and imaginary parts of the inertial mode $\boldsymbol{u}_{10}$ given by (3.16)-(3.18),

$$
\begin{aligned}
\boldsymbol{Q}_{c}= & {\left[\frac{\tau \mathscr{E}^{2} \sqrt{1-\tau^{2}}\left(1-\mathscr{E}^{2}-\eta^{2}\right) \cos \phi}{\sqrt{\eta^{2}+\tau^{2} \mathscr{E}^{2}}}\right] \hat{\boldsymbol{\eta}}+[\eta \tau \sin \phi] \hat{\boldsymbol{\phi}} } \\
& +\left[\frac{\eta \sqrt{\eta^{2}+\mathscr{E}^{2}}\left(1-\mathscr{E}^{2}+\mathscr{E}^{2} \tau^{2}\right) \cos \phi}{\sqrt{\eta^{2}+\tau^{2} \mathscr{E}^{2}}}\right] \hat{\boldsymbol{\tau}}, \\
\boldsymbol{Q}_{s}= & -\left[\frac{\tau \mathscr{E}^{2} \sqrt{1-\tau^{2}}\left(1-\mathscr{E}^{2}-\eta^{2}\right) \sin \phi}{\sqrt{\eta^{2}+\tau^{2} \mathscr{E}^{2}}}\right] \hat{\boldsymbol{\eta}}+[\eta \tau \cos \phi] \hat{\boldsymbol{\phi}} \\
& -\left[\frac{\eta \sqrt{\eta^{2}+\mathscr{E}^{2}}\left(1-\mathscr{E}^{2}+\mathscr{E}^{2} \tau^{2}\right) \sin \phi}{\sqrt{\eta^{2}+\tau^{2} \mathscr{E}^{2}}}\right] \hat{\boldsymbol{\tau}} .
\end{aligned}
$$

It is worth noting that the asymptotic expression (3.53), valid only for $E \ll 1$, represents an oscillatory flow (i.e. its amplitude $|\boldsymbol{u}|$ is a function of time $t$ ) within a spheroidal cavity of arbitrary eccentricity $0<\mathscr{E}<1$, driven by latitudinal libration 


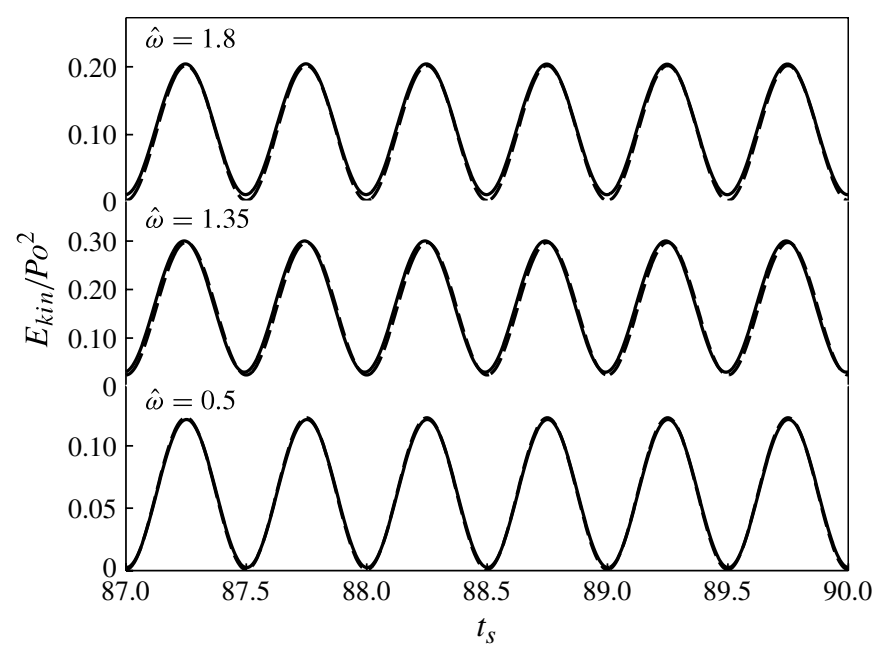

FIGURE 1. Scaled kinetic energy $E_{\text {kin }}$ of the librationally driven flows plotted as a function of $t_{s}=t / T$, where $T=2 \pi / \hat{\omega}$ for $\beta=0.005, E=5 \times 10^{-5}$ and $\mathscr{E}=0.5$. Three numerical simulations for $\hat{\omega}=0.5,1.35,1.8$ are shown as solid lines; the corresponding asymptotic solutions (dashed lines), computed from the analytical expression (3.57), are also presented.

with its frequency $\hat{\omega}$ satisfying

$$
\left|\hat{\omega}-\frac{2}{\left(2-\mathscr{E}^{2}\right)}\right| \gg O\left(E^{1 / 2}\right) .
$$

To measure the strength of a librating flow, we may introduce the kinetic energy density, $E_{k i n}$, defined as

$$
E_{k i n}(t)=\frac{1}{2 V} \int_{V}|\boldsymbol{u}|^{2} \mathrm{~d} V,
$$

where $V$ is the volume of the spheroidal cavity. The leading order of $E_{k i n}$ can be computed using the analytical expression (3.53):

$$
\begin{aligned}
E_{k i n}(t)= & \frac{2 P o^{2}\left(1-\mathscr{E}^{2}\right)\left(2-\mathscr{E}^{2}\right)}{5\left[\hat{\omega}^{2}\left(2-\mathscr{E}^{2}\right)^{2}-4\right]^{2}} \\
& \times\left\{\left[\hat{\omega}^{2}\left(2-\mathscr{E}^{2}\right)-2\right]^{2} \sin ^{2} \hat{\omega} t+\hat{\omega}^{2} \mathscr{E}^{4} \cos ^{2} \hat{\omega} t\right\}+O\left(E^{1 / 2}\right) .
\end{aligned}
$$

Figure 1 shows the kinetic energy $E_{k i n}(t)$, calculated directly from the asymptotic expression (3.57), as a function of time for several different frequencies for $P o=0.005$ and $\mathscr{E}=0.5$ at $E=5 \times 10^{-5}$. The profile of the flow, computed from the analytical solution (3.53), is displayed in figure $2(b, d)$. Its detail will be discussed in $\S 4$ along with the result of the corresponding numerical simulation.

\subsection{Resonant flow with an active boundary layer}

Prior to the asymptotic analysis for resonant flow, we make several important observations in the expressions (3.39) and (3.53). First, the amplitude of the flow $|\boldsymbol{u}| \rightarrow \infty$ when the libration frequency $\hat{\omega}$ approaches $\omega_{10}=2 \sigma_{10}=2 /\left(2-\mathscr{E}^{2}\right)$. Hence the asymptotic solution (3.53) breaks down as $\hat{\omega} \rightarrow \omega_{10}$, suggesting resonance with latitudinal libration. Second, the two solvability conditions (3.27) and (3.28) neglecting 

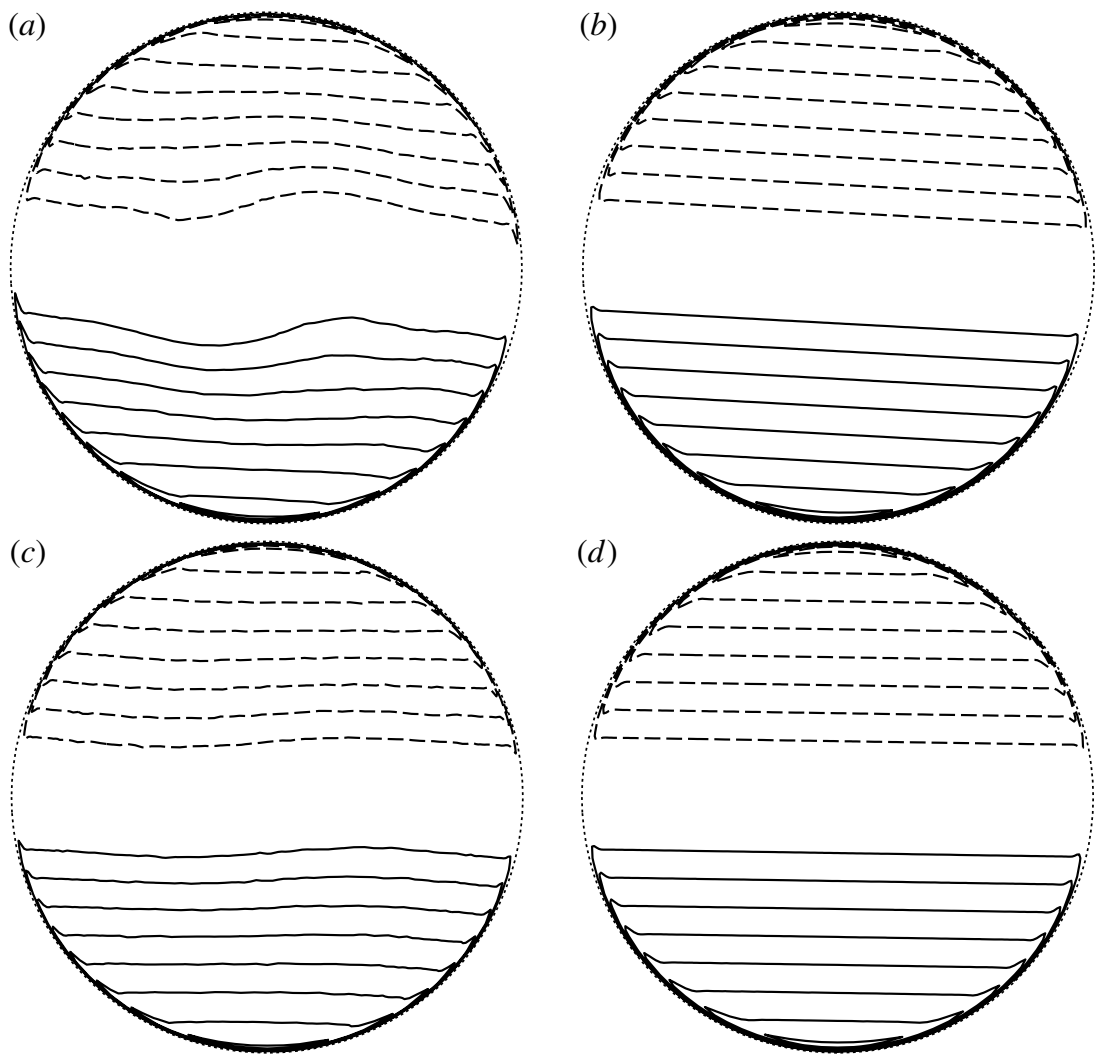

FIGURE 2. Contours of the flow component $\hat{\boldsymbol{\tau}} \cdot \boldsymbol{u}$ at the equatorial $z=0$ plane obtained from both numerical simulation and asymptotic solution for non-resonant flows: $(a) \hat{\omega}=1.35$ (numerical); (b) $\hat{\omega}=1.35$ (asymptotic); (c) $\hat{\omega}=1.8$ (numerical); $(d) \hat{\omega}=1.8$ (asymptotic). Other parameters of the solutions are $P o=0.005$ and $E=5 \times 10^{-5}$ with eccentricity $\mathscr{E}=0.5$. Solid contours are for $(\hat{\boldsymbol{\tau}} \cdot \boldsymbol{u}>0)$ while dashed contours for $(\hat{\boldsymbol{\tau}} \cdot \boldsymbol{u}<0)$.

the effect of the boundary-layer flux cannot determine the values of $A_{10}$ and $B_{10}$ at the resonance. It follows that the four integrals in (3.21) and (3.22), which are neglected in deriving (3.27) and (3.28), must be included. Third, it is vital to recognize that $A_{10} \rightarrow \mathrm{i} B_{10}$ as $\hat{\omega} \rightarrow \omega_{10}$. In the expression (3.39) for $A_{10}$ and $B_{10}$, we can readily show that

$$
\left[\hat{\omega}^{2}\left(2-\mathscr{E}^{2}\right)-2\right] \rightarrow \hat{\omega} \mathscr{E}^{2} \text { as } \hat{\omega} \rightarrow \omega_{10},
$$

signalling that $A_{10}$ is no longer independent of $B_{10}$ at the resonance. This feature is also reflected in the two solvability conditions (3.21) and (3.22), which become identical in the limit $\hat{\omega} \rightarrow \omega_{10}$. Finally, a consequence of $A_{10} \rightarrow \mathrm{i} B_{10}$ as $\hat{\omega} \rightarrow \omega_{10}$ is that the asymptotic solution (3.53) is no longer oscillatory: it becomes an azimuthally, retrogradely travelling wave in the form of $\boldsymbol{u}(\eta, \tau, \phi, t) \sim \boldsymbol{u}(\eta, \tau) \exp \left[\mathrm{i}\left(\phi+\omega_{10} t\right)\right]$. More precisely, the interior part of the asymptotic solution (3.53) becomes proportional to $\boldsymbol{u}_{10} \exp \left[\mathrm{i}\left(\omega_{10} t\right)\right]$, which satisfies (3.19) and (3.20) exactly, implying that the spheroidal inertial mode $\boldsymbol{u}_{10}$ with the frequency $\omega_{10}=2 /\left(2-\mathscr{E}^{2}\right)$ can resonate with latitudinal libration.

The above observations suggest that, after making use of the fact that $A_{n k}=0$ and $B_{n k}=0$ except for $n=1$ and $k=0$, the asymptotic expansions (3.3) and (3.4) at the 
resonance may be rewritten as

$$
\begin{aligned}
\boldsymbol{u} & =\left[\left(A_{10} \boldsymbol{u}_{10}+\text { c.c. }\right)+\widehat{\boldsymbol{u}}_{s}+\widetilde{\boldsymbol{u}}_{s}\right] \sin \hat{\omega} t+\left[\left(B_{10} \boldsymbol{u}_{10}+\text { c.c. }\right)+\widehat{\boldsymbol{u}}_{c}+\widetilde{\boldsymbol{u}}_{c}\right] \cos \hat{\omega} t \\
& =\frac{1}{2}\left[-2 i \mathscr{A} \boldsymbol{u}_{10}+\left(\widehat{\boldsymbol{u}}_{c}-\mathrm{i} \widehat{\boldsymbol{u}}_{s}\right)+\left(\widetilde{\boldsymbol{u}}_{c}-\mathrm{i} \widetilde{\boldsymbol{u}}_{s}\right)\right] \mathrm{e}^{\mathrm{i} \hat{\omega} t}+\text { c.c. }
\end{aligned}
$$

Here we have assumed that $\mathscr{A}=A_{10}$ and $B_{10}=-\mathrm{i} \mathscr{A}$, with $\mathscr{A}$ being an unknown complex amplitude to be determined. The pressure $p$ can be rewritten in a similar way,

$$
\begin{aligned}
p & =\left[\left(A_{10} p_{10}+\text { c.c. }\right)+\widehat{p}_{s}+\widetilde{p}_{s}\right] \sin \hat{\omega} t+\left[\left(B_{10} p_{10}+\text { c.c. }\right)+\widehat{p}_{c}+\widetilde{p}_{c}\right] \cos \hat{\omega} t \\
& =\frac{1}{2}\left[-2 i \mathscr{A} p_{10}+\left(\widehat{p}_{c}-\mathrm{i} \widehat{p}_{s}\right)+\left(\widetilde{p}_{c}-\mathrm{i} \widetilde{p}_{s}\right)\right] \mathrm{e}^{\mathrm{i} \hat{\omega} t}+\text { c.c. }
\end{aligned}
$$

It is the effect of the viscous boundary layer, which now plays an active role, that determines the amplitude $\mathscr{A}$ of librationally driven flow at the resonance.

As $\hat{\omega} \rightarrow \omega_{10}=2 /\left(2-\mathscr{E}^{2}\right)$, the two solvability conditions, (3.21) and (3.22), are merged into a single condition:

$$
\begin{aligned}
& \hat{\omega} \int_{V} \boldsymbol{u}_{10}^{*} \cdot\left(\hat{\omega} \widehat{\boldsymbol{u}}_{s}+2 \hat{z} \times \widehat{\boldsymbol{u}}_{c}+\nabla \widehat{p}_{c}\right) \mathrm{d} V-\mathrm{i} \omega_{10} \int_{V} \boldsymbol{u}_{10}^{*} \cdot\left(-\hat{\omega} \widehat{\boldsymbol{u}}_{c}+2 \hat{z} \times \widehat{\boldsymbol{u}}_{s}+\nabla \widehat{p}_{s}\right) \mathrm{d} V \\
& \quad=P o \int_{V} \boldsymbol{u}_{10}^{*} \cdot\left[\hat{\omega}^{2} \boldsymbol{r} \times \hat{\boldsymbol{x}}-\mathrm{i} \omega_{10} \boldsymbol{r} \times(\hat{z} \times \hat{\boldsymbol{x}})\right] \mathrm{d} V
\end{aligned}
$$

where $\boldsymbol{u}_{10}^{*}$ denotes the complex conjugate of $\boldsymbol{u}_{10}$. Note that (3.61) does not explicitly contain the unknown amplitude $\mathscr{A}$. It is the secondary interior flow, $\widehat{\boldsymbol{u}}_{c}$ and $\widehat{\boldsymbol{u}}_{s}$, that implicitly links the solvability condition (3.61) with $\mathscr{A}$ through the boundary-layer flux. After some rearrangement, the solvability condition can be written as

$$
\begin{aligned}
& \int_{V} \boldsymbol{u}_{10}^{*} \cdot\left[\mathrm{i} \omega_{10}\left(\widehat{\boldsymbol{u}}_{c}-\mathrm{i} \widehat{\boldsymbol{u}}_{s}\right)+2 \hat{z} \times\left(\widehat{\boldsymbol{u}}_{c}-\mathrm{i} \widehat{\boldsymbol{u}}_{s}\right)+\nabla\left(\widehat{p}_{c}-\mathrm{i} \widehat{p}_{s}\right)\right] \mathrm{d} V \\
& =P o \int_{V} \boldsymbol{u}_{10}^{*} \cdot\left[\omega_{10} \boldsymbol{r} \times \hat{\boldsymbol{x}}-\mathrm{i} \boldsymbol{r} \times(\hat{\boldsymbol{z}} \times \hat{\boldsymbol{x}})\right] \mathrm{d} V .
\end{aligned}
$$

Upon making use of $\boldsymbol{\nabla} \cdot \boldsymbol{u}_{10}^{*}=0$ and $\hat{\boldsymbol{n}} \cdot \boldsymbol{u}_{10}^{*}=0$ on the bounding surface $\mathscr{S}$, the condition (3.62) reduces to

$$
\begin{gathered}
\int_{0}^{2 \pi} \int_{-1}^{1}\left[p_{10}^{*} \hat{\boldsymbol{n}} \cdot\left(\widehat{\boldsymbol{u}}_{c}-\mathrm{i} \widehat{\boldsymbol{u}}_{s}\right)\right]_{\mathscr{S}} \sqrt{1-\mathscr{E}^{2}+\tau^{2} \mathscr{E}^{2}} \mathrm{~d} \tau \mathrm{d} \phi \\
\quad=P o \int_{V} \boldsymbol{u}_{10}^{*} \cdot\left[\left(\frac{2}{2-\mathscr{E}^{2}}\right) \boldsymbol{r} \times \hat{\boldsymbol{x}}-\mathrm{i} \boldsymbol{r} \times(\hat{z} \times \hat{\boldsymbol{x}})\right] \mathrm{d} V,
\end{gathered}
$$

where $\left[p_{10}^{*}\right]_{\mathscr{S}}$ denotes the evaluation of $p_{10}^{*}$, the complex conjugate of $p_{10}$, at the bounding surface $\mathscr{S}$, and $\left[\hat{\boldsymbol{n}} \cdot\left(\hat{\boldsymbol{u}}_{c}-\mathrm{i} \hat{\boldsymbol{u}}_{s}\right)\right]_{\mathscr{S}}$ represents the normal flux at the outer edge of the viscous boundary layer. In contrast to the non-resonant case, it is now imperative to derive an asymptotic expression for the mass flux, which is usually cumbersome and lengthy. In order to obtain the amplitude $\mathscr{A}$ at the resonance, the following three steps are required: (i) derive the viscous boundary-layer solution $\left(\widetilde{\boldsymbol{u}}_{c}-\mathrm{i} \widetilde{\boldsymbol{u}}_{s}\right)$; (ii) obtain an expression for the boundary-layer flux $\left[\hat{\boldsymbol{n}} \cdot\left(\hat{\boldsymbol{u}}_{c}-\mathrm{i} \hat{\boldsymbol{u}}_{s}\right)\right]_{\mathscr{S}}$; and (iii) carry out the resulting integrations in (3.63). It is worth mentioning that, because the resonant solution is in the form of an azimuthally travelling wave with constant amplitude, the boundary-layer solution $\left(\widetilde{\boldsymbol{u}}_{c}-\mathbf{i} \widetilde{\boldsymbol{u}}_{s}\right)$ is actually simpler than that for the non-resonant case. 
First, consider the viscous boundary-layer flow $\left(\widetilde{\boldsymbol{u}}_{c}-\mathrm{i} \widetilde{\boldsymbol{u}}_{s}\right)$ whose governing equation, a fourth-order differential equation, can be derived from (3.23) and (3.24):

$$
\left(\frac{\partial^{2}}{\partial \xi^{2}}-\frac{2 \mathrm{i}}{2-\mathscr{E}^{2}}\right)^{2}\left(\widetilde{\boldsymbol{u}}_{c}-\mathrm{i} \widetilde{\boldsymbol{u}}_{s}\right)+\left(\frac{2 \tau}{\sqrt{1-\mathscr{E}^{2}+\mathscr{E}^{2} \tau^{2}}}\right)^{2}\left(\widetilde{\boldsymbol{u}}_{c}-\mathrm{i} \widetilde{\boldsymbol{u}}_{s}\right)=0 .
$$

This can be solved subject to the following four boundary conditions that ensure the tangential velocity vanishes at the bounding surface of the spheroidal cavity:

$$
\begin{aligned}
{\left[\widetilde{\boldsymbol{u}}_{c}-\mathrm{i} \widetilde{\boldsymbol{u}}_{s}\right]_{\xi=0}=} & \widetilde{\boldsymbol{V}}_{0} \\
= & \frac{3\left(2-\mathscr{E}^{2}\right) \mathscr{A}}{2\left(1-\mathscr{E}^{2}\right)^{1 / 2}}\left[-\left(1-\mathscr{E}^{2}+\tau^{2} \mathscr{E}^{2}\right)^{1 / 2} \hat{\boldsymbol{\tau}}+\mathrm{i} \tau \hat{\boldsymbol{\phi}}\right] \mathrm{e}^{\mathrm{i}\left[\phi+2 t /\left(2-\mathscr{E}^{2}\right)\right]}, \\
{\left[\frac{\partial^{2}}{\partial \xi^{2}}\left(\widetilde{\boldsymbol{u}}_{c}-\mathrm{i} \widetilde{\boldsymbol{u}}_{s}\right)\right]_{\xi=0}=} & \frac{2 \mathrm{i} \widetilde{\boldsymbol{V}}_{0}}{\left(2-\widetilde{\mathscr{E}}^{2}\right)}+\left(\frac{2 \tau}{\sqrt{1-\mathscr{E}^{2}+\mathscr{E}^{2} \tau^{2}}}\right) \hat{\boldsymbol{n}} \times \widetilde{\boldsymbol{V}}_{0}, \\
& {\left[\frac{\partial^{2}}{\partial \xi^{2}}\left(\widetilde{\boldsymbol{u}}_{c}-\mathrm{i} \widetilde{\boldsymbol{u}}_{s}\right)\right]_{\xi=\infty}=0 . }
\end{aligned}
$$

The boundary-layer solution that satisfies both the fourth-order equation (3.64) and the four boundary conditions is given by

$$
\begin{aligned}
\left(\widetilde{\boldsymbol{u}}_{c}-\mathrm{i} \widetilde{\boldsymbol{u}}_{s}\right)= & \frac{3\left(2-\mathscr{E}^{2}\right) \mathscr{A}}{4\left(1-\mathscr{E}^{2}\right)^{1 / 2}}\left\{\left(\tau-\sqrt{1-\mathscr{E}^{2}+\mathscr{E}^{2} \tau^{2}}\right)(\hat{\boldsymbol{\tau}}+\mathrm{i} \hat{\boldsymbol{\phi}}) \mathrm{e}^{\gamma^{+} \xi}\right. \\
& \left.+\left(\tau+\sqrt{1-\mathscr{E}^{2}+\mathscr{E}^{2} \tau^{2}}\right)(-\hat{\boldsymbol{\tau}}+\mathrm{i} \hat{\boldsymbol{\phi}}) \mathrm{e}^{\gamma^{-} \xi}\right\} \mathrm{e}^{\mathrm{i}\left[\phi+2 t /\left(2-\mathscr{E}^{2}\right)\right]},
\end{aligned}
$$

where $\gamma^{ \pm}$is a function of $\tau$,

$$
\gamma^{ \pm}=-\left(1+i s^{ \pm}\right)\left[\frac{\mid \tau\left(2-\mathscr{E}^{2}\right) \pm \sqrt{1-\mathscr{E}^{2}+\mathscr{E}^{2} \tau^{2} \mid}}{\left(2-\mathscr{E}^{2}\right) \sqrt{1-\mathscr{E}^{2}+\mathscr{E}^{2} \tau^{2}}}\right]^{1 / 2},
$$

with $s^{ \pm}$denoting a sign function defined as

$$
s^{ \pm}=\frac{\tau\left(2-\mathscr{E}^{2}\right) \pm \sqrt{1-\mathscr{E}^{2}+\mathscr{E}^{2} \tau^{2}}}{\left|\tau\left(2-\mathscr{E}^{2}\right) \sqrt{1-\mathscr{E}^{2}+\mathscr{E}^{2} \tau^{2}}\right|} .
$$

In (3.66), the outer edge of the thin viscous boundary layer is defined by taking the limit $\xi \rightarrow \infty$.

Second, the normal component of the mass flux at the outer edge of the viscous boundary layer, which links the boundary-layer solution to the secondary interior flow, is given by (Greenspan 1968),

$$
\left[\hat{\boldsymbol{n}} \cdot\left(\widehat{\boldsymbol{u}}_{c}-\mathrm{i} \widehat{\boldsymbol{u}}_{s}\right)\right]_{\mathscr{S}}=E^{1 / 2} \int_{0}^{\infty} \hat{\boldsymbol{n}} \cdot \nabla \times\left[\hat{\boldsymbol{n}} \times\left(\widetilde{\boldsymbol{u}}_{c}-\mathrm{i} \widetilde{\boldsymbol{u}}_{s}\right)\right] \mathrm{d} \xi,
$$

providing the asymptotic matching condition required for evaluating the solvability condition (3.63). With oblate spheroidal coordinates, (3.69) can be written as

$$
\begin{aligned}
{\left[\hat{\boldsymbol{n}} \cdot\left(\widehat{\boldsymbol{u}}_{c}-\mathrm{i} \widehat{\boldsymbol{u}}_{s}\right)\right]_{\mathscr{S}}=} & \frac{E^{1 / 2}}{\sqrt{1-\mathscr{E}^{2}+\mathscr{E}^{2} \tau^{2}}} \int_{0}^{\infty}\left\{\frac{\partial}{\partial \phi}\left[\frac{1-\mathscr{E}^{2}+\mathscr{E}^{2} \tau^{2}}{1-\tau^{2}} \hat{\boldsymbol{\phi}} \cdot\left(\widetilde{\boldsymbol{u}}_{c}-\mathrm{i} \widetilde{\boldsymbol{u}}_{s}\right)\right]\right. \\
& \left.+\frac{\partial}{\partial \tau}\left[\sqrt{\left(1-\mathscr{E}^{2}+\mathscr{E}^{2} \tau^{2}\right)\left(1-\tau^{2}\right)} \hat{\boldsymbol{\tau}} \cdot\left(\widetilde{\boldsymbol{u}}_{c}-\mathrm{i} \widetilde{\boldsymbol{u}}_{s}\right)\right]\right\} \mathrm{d} \xi .
\end{aligned}
$$


On inserting the boundary-layer solution (3.66) into (3.70) and then performing the integration over $\xi$, we obtain an expression for the mass flux at the outer edge of the viscous boundary layer:

$$
\begin{aligned}
& {\left[\hat{\boldsymbol{n}} \cdot\left(\widehat{\boldsymbol{u}}_{c}-\mathrm{i} \widehat{\boldsymbol{u}}_{s}\right)\right]_{\mathscr{S}}=-\frac{E^{1 / 2}}{\sqrt{1-\mathscr{E}^{2}+\mathscr{E}^{2} \tau^{2}}}\left[\frac{3\left(2-\mathscr{E}^{2}\right) \mathscr{A}}{4 \sqrt{1-\mathscr{E}^{2}}}\right] \mathrm{e}^{\mathrm{i}\left[\phi+2 t /\left(2-\mathscr{E}^{2}\right)\right]}} \\
& \quad \times\left\{\frac{1-\mathscr{E}^{2}+\mathscr{E}^{2} \tau^{2}}{\sqrt{1-\tau^{2}}}\left[\frac{-\tau+\sqrt{1-\mathscr{E}^{2}+\mathscr{E}^{2} \tau^{2}}}{\gamma^{+}}-\frac{\tau+\sqrt{1-\mathscr{E}^{2}+\mathscr{E}^{2} \tau^{2}}}{\gamma^{-}}\right]\right. \\
& \quad+\frac{\mathrm{d}}{\mathrm{d} \tau}\left[\frac{\sqrt{\left(1-\mathscr{E}^{2}+\mathscr{E}^{2} \tau^{2}\right)\left(1-\tau^{2}\right)}\left(\tau-\sqrt{\left.1-\mathscr{E}^{2}+\mathscr{E}^{2} \tau^{2}\right)}\right.}{\gamma^{+}}\right] \\
& \left.\quad-\frac{\mathrm{d}}{\mathrm{d} \tau}\left[\frac{\sqrt{\left(1-\mathscr{E}^{2}+\mathscr{E}^{2} \tau^{2}\right)\left(1-\tau^{2}\right)}\left(\tau+\sqrt{1-\mathscr{E}^{2}+\mathscr{E}^{2} \tau^{2}}\right)}{\gamma^{-}}\right]\right\} .
\end{aligned}
$$

In the process of computing the normal flux from the viscous boundary layer, it should be noted that both $\gamma^{+}$and $\gamma^{-}$are functions of $\tau$.

Third, upon substituting the pressure $\left(p_{10}^{*}\right)_{\mathscr{S}}$ given by (3.15) and the mass flux $\left[\hat{\boldsymbol{n}} \cdot\left(\widehat{\boldsymbol{u}}_{c}-\mathrm{i} \widehat{\boldsymbol{u}}_{s}\right)\right]_{\mathscr{S}}$ given by (3.71) into the solvability condition (3.63), we can derive an equation for the amplitude $\mathscr{A}$ :

$$
-\frac{9 \pi E^{1 / 2} \mathscr{A}\left(2-\mathscr{E}^{2}\right)^{3 / 2}}{4}=\frac{P o}{\mathscr{I}_{r}+\mathrm{i} \mathscr{I}_{i}} \int_{V} \boldsymbol{u}_{10}^{*} \cdot\left[\left(\frac{2}{2-\mathscr{E}^{2}}\right) \boldsymbol{r} \times \hat{\boldsymbol{x}}-\mathrm{i} \boldsymbol{r} \times(\hat{z} \times \hat{\boldsymbol{x}})\right] \mathrm{d} V .
$$

Here $\mathscr{I}_{r}$ and $\mathscr{I}_{i}$ denote the following two complicated integrals:

$$
\begin{gathered}
\mathscr{I}_{r}=\int_{-1}^{+1} \frac{\left(1-\mathscr{E}^{2}+\mathscr{E}^{2} \tau^{2}\right)^{3 / 4}\left(-\tau+\sqrt{1-\mathscr{E}^{2}+\mathscr{E}^{2} \tau^{2}}\right)\left(1-2 \tau^{2}+\tau \sqrt{1-\mathscr{E}^{2}+\mathscr{E}^{2} \tau^{2}}\right)}{\left|\left(2-\mathscr{E}^{2}\right) \tau^{2}+\sqrt{1-\mathscr{E}^{2}+\mathscr{E}^{2} \tau^{2}}\right|^{1 / 2}} \mathrm{~d} \tau, \\
\mathscr{I}_{i}=\int_{-1}^{+1} \frac{\left(1-\mathscr{E}^{2}+\mathscr{E}^{2} \tau^{2}\right)^{3 / 4}\left(\tau-\sqrt{1-\mathscr{E}^{2}+\mathscr{E}^{2} \tau^{2}}\right)\left(1-2 \tau^{2}+\tau \sqrt{1-\mathscr{E}^{2}+\mathscr{E}^{2} \tau^{2}}\right)}{\left|\left(2-\mathscr{E}^{2}\right) \tau^{2}+\sqrt{1-\mathscr{E}^{2}+\mathscr{E}^{2} \tau^{2}}\right|^{3 / 2}} \mathrm{~d} \tau .
\end{gathered}
$$

The volume integration on the right-hand side of (3.72), after using the expression $\boldsymbol{u}_{10}$ given by (3.16)-(3.18), can be readily carried out, giving rise to

$$
\int_{V} \boldsymbol{u}_{10}^{*} \cdot\left[\left(\frac{2}{2-\mathscr{E}^{2}}\right) \boldsymbol{r} \times \hat{\boldsymbol{x}}-\mathrm{i} \boldsymbol{r} \times(\hat{z} \times \hat{\boldsymbol{x}})\right] \mathrm{d} V=\frac{2 \pi\left(1-\mathscr{E}^{2}\right)^{1 / 2} \mathscr{E}^{2}}{5} .
$$

It follows that, after solving (3.72), the amplitude $\mathscr{A}$ is given by

$$
\mathscr{A}=\frac{P o \mathscr{E}^{2}}{E^{1 / 2}}\left[\frac{\mathscr{I}_{r}-\mathrm{i} \mathscr{I}_{i}}{\mathscr{I}_{r}^{2}+\mathscr{I}_{i}^{2}}\right] \frac{8\left(1-\mathscr{E}^{2}\right)^{1 / 2}}{45\left(2-\mathscr{E}^{2}\right)^{3 / 2}} .
$$

A careful manipulation is, however, required for evaluating $\mathscr{I}_{r}$ and $\mathscr{I}_{i}$. This is because there exist two critical co-latitudes $\tau_{c}= \pm 1 / \sqrt{4-\mathscr{E}^{2}}$ at which the boundary-layer solution breaks down as it thickens to $O\left(E^{2 / 5}\right)$, as discussed by Roberts \& Stewartson (1965). However, it is generally believed that the effect of the singularities is weak and insignificant because the mass flux from the critical regions is much smaller than that 
from the rest of the boundary layer (see e.g. Roberts \& Stewartson 1965; Busse 1968; Tilgner \& Busse 2001).

We are now in a position to write an asymptotic expression for the resonant flow in a spheroidal cavity of arbitrary eccentricity driven by latitudinal libration for an asymptotically small $E$. By inserting the expressions for $\mathscr{A}$ and $\widetilde{\boldsymbol{u}}_{c}+\mathrm{i} \widetilde{\boldsymbol{u}}_{s}$ into the expansion (3.59), we obtain the asymptotic solution for the resonant flow:

$$
\begin{aligned}
& \boldsymbol{u}=\left(\frac{P_{o} \mathscr{E}^{2}}{E^{1 / 2}}\right)\left[\frac{2}{15 \sqrt{\left(1-\mathscr{E}^{2}\right)\left(2-\mathscr{E}^{2}\right)}}\right]\left(\frac{\mathscr{I}_{r}-\mathrm{i} \mathscr{I}_{i}}{\mathscr{I}_{r}^{2}+\mathscr{I}_{i}^{2}}\right) \\
& \times\left\{\left[\frac{\tau \mathscr{E}^{2} \sqrt{1-\tau^{2}}\left(1-\mathscr{E}^{2}-\eta^{2}\right)}{\sqrt{\eta^{2}+\mathscr{E}^{2} \tau^{2}}} \hat{\boldsymbol{\eta}}+\frac{\eta \sqrt{\eta^{2}+\mathscr{E}^{2}}\left(1-\mathscr{E}^{2}+\mathscr{E}^{2} \tau^{2}\right)}{\sqrt{\eta^{2}+\mathscr{E}^{2} \tau^{2}}} \hat{\boldsymbol{\tau}}-\mathrm{i} \eta \tau \hat{\boldsymbol{\phi}}\right]\right. \\
& +\frac{\sqrt{1-\mathscr{E}^{2}}}{2}\left[\left(\tau-\sqrt{1-\mathscr{E}^{2}+\mathscr{E}^{2} \tau^{2}}\right)(\hat{\boldsymbol{\tau}}+\mathrm{i} \hat{\boldsymbol{\phi}}) \mathrm{e}^{\gamma^{+} \xi}\right. \\
& \left.\left.+\left(\tau+\sqrt{1-\mathscr{E}^{2}+\mathscr{E}^{2} \tau^{2}}\right)(-\hat{\boldsymbol{\tau}}+\mathrm{i} \hat{\boldsymbol{\phi}}) \mathrm{e}^{\gamma^{-} \xi}\right]\right\} \mathrm{e}^{\mathrm{i}\left[\phi+2 t /\left(2-\mathscr{E}^{2}\right)\right]}+\text { c.c. }
\end{aligned}
$$

Its kinetic energy $E_{k i n}$ at leading order is simply given by

$$
E_{k i n}=\left(\frac{P o^{2} \mathscr{E}^{4}}{E}\right) \frac{4}{1125\left(\mathscr{I}_{r}^{2}+\mathscr{I}_{i}^{2}\right)} .
$$

For highly flattened spheroids with large values of eccentricity $\mathscr{E}$, the integrals $\mathscr{I}_{r}$ and $\mathscr{I}_{i}$ have to be evaluated numerically.

For moderately small eccentricity with $\mathscr{E}^{2} \ll 1$, which represents the case for the majority of known planets, relatively simple asymptotic expressions for $\mathscr{I}_{r}$ and $\mathscr{I}_{i}$ can be derived:

$$
\begin{gathered}
\mathscr{I}_{r}=-\frac{2}{35}(19-9 \sqrt{3})-\mathscr{E}^{2}\left[\frac{4}{55}\left(\frac{1}{9}+6 \sqrt{3}\right)\right]+O\left(\mathscr{E}^{4}\right), \\
\mathscr{I}_{i}=\frac{2}{5}(7-3 \sqrt{3})+\mathscr{E}^{2}\left[\frac{4}{105}(52-3 \sqrt{3})\right]+O\left(\mathscr{E}^{4}\right) .
\end{gathered}
$$

Further details about the above integrals are provided in the Appendix. In this case, an explicitly analytical solution becomes possible:

$$
\begin{aligned}
\boldsymbol{u}= & \left(\frac{P o \mathscr{E}^{2}}{E^{1 / 2}}\right)\left[\frac{2}{15 \sqrt{\left(1-\mathscr{E}^{2}\right)\left(2-\mathscr{E}^{2}\right)}}\right] \\
& \times\left\{\left[\frac{2(-19+9 \sqrt{3})}{35}-\frac{\mathrm{i} 2(7-3 \sqrt{3})}{3}\right]-\mathscr{E}^{2}\left[\frac{4(1+54 \sqrt{3})}{495}+\frac{\mathrm{i} 4(52-3 \sqrt{3})]}{105}\right]\right\} \\
& \times\left[\frac{17312}{1225}-\frac{384 \sqrt{3}}{49}+\mathscr{E}^{2}\left(\frac{183424}{17325}-\frac{8576 \sqrt{3}}{1925}\right)\right]^{-1} \\
& \times\left\{\left[\frac{\tau \mathscr{E}^{2} \sqrt{1-\tau^{2}}\left(1-\mathscr{E}^{2}-\eta^{2}\right)}{\sqrt{\eta^{2}+\mathscr{E}^{2} \tau^{2}}} \hat{\boldsymbol{\eta}}+\frac{\eta \sqrt{\eta^{2}+\mathscr{E}^{2}}\left(1-\mathscr{E}^{2}+\mathscr{E}^{2} \tau^{2}\right)}{\sqrt{\eta^{2}+\mathscr{E}^{2} \tau^{2}}} \hat{\boldsymbol{\tau}}-\mathrm{i} \eta \tau \hat{\boldsymbol{\phi}}\right]\right. \\
& +\frac{\sqrt{1-\mathscr{E}^{2}}\left[\left(\tau-\sqrt{1-\mathscr{E}^{2}+\mathscr{E}^{2} \tau^{2}}\right)(\hat{\boldsymbol{\tau}}+\mathrm{i} \hat{\boldsymbol{\phi}}) \mathrm{e}^{\gamma^{+} \xi}\right.}{2}\left[\begin{array}{l}
\mathrm{i} \\
+
\end{array}\right. \\
& \left.\left.\left(\tau+\sqrt{1-\mathscr{E}^{2}+\mathscr{E}^{2} \tau^{2}}\right)(-\hat{\boldsymbol{\tau}}+\mathrm{i} \hat{\boldsymbol{\phi}}) \mathrm{e}^{\gamma^{-} \xi}\right]\right\} \mathrm{e}^{\mathrm{i}\left[\phi+2 t /\left(2-\mathscr{E}^{2}\right)\right]}+\text { c.c. },
\end{aligned}
$$



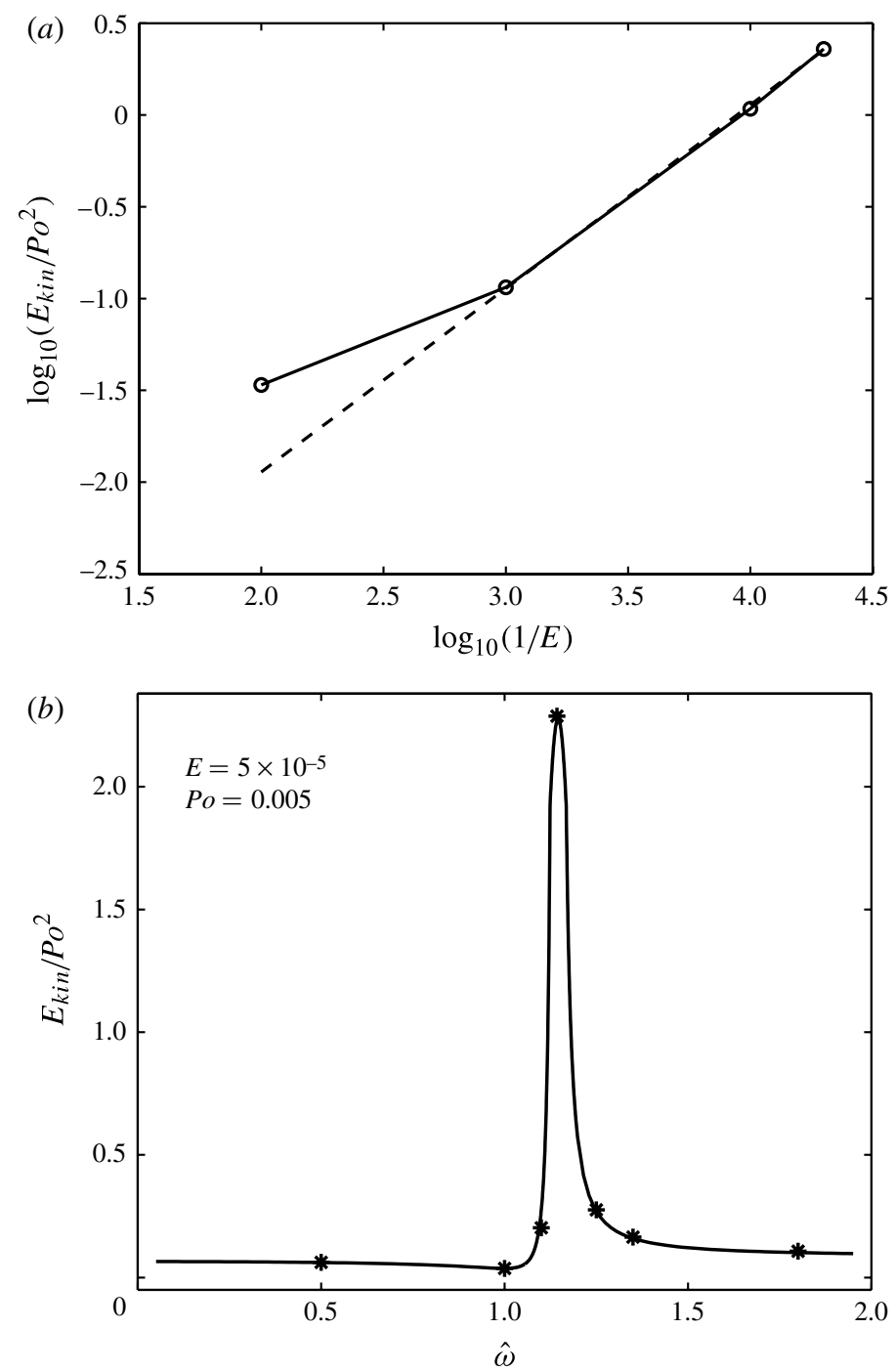

FIgURE 3. (a) Scaled kinetic energy $E_{k i n} / P o^{2}$ plotted as a function of $E^{-1}$ at the resonance frequency $\hat{\omega}=8 / 7$ for $\mathscr{E}=0.5$. The dashed line is computed directly from the asymptotic expression (3.77), while the solid line represents the result of direct numerical simulation at four Ekman numbers $E=10^{-2}, 10^{-3}, 10^{-4}$ and $5 \times 10^{-5}$ with $P o=0.005$ and $\mathscr{E}=0.5$. (b) Scaled kinetic energy $E_{k i n} / P o^{2}$ plotted as a function of the libration frequency $\hat{\omega}$ for $E=5 \times 10^{-5}$ and $\mathscr{E}=0.5$. The solid line is based on the result of the asymptotic formulae (3.57) and (3.77), while the $*$ symbols represent the results of direct numerical simulation.

representing an asymptotic solution for the resonant flow, driven by latitudinal libration, within a spheroidal cavity with $\mathscr{E}^{2} \ll 1$. The leading-order kinetic energy of the resonant flow is

$$
E_{k i n}=\left(\frac{4 P o^{2} \mathscr{E} 4}{1125 E}\right)\left[\frac{17312}{1225}-\frac{384 \sqrt{3}}{49}+\mathscr{E}^{2}\left(\frac{183424}{17325}-\frac{8576 \sqrt{3}}{1925}\right)\right]^{-1},
$$

which is valid when $\mathscr{E}^{2} \ll 1$ and $E \ll 1$. 


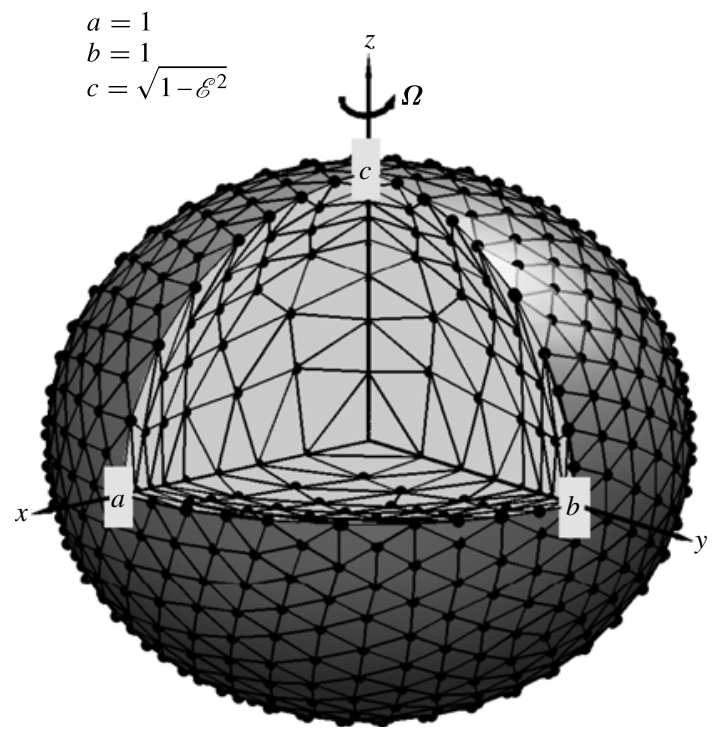

FIGURE 4. A schematic of the spheroidal mesh with $\mathscr{E}=0.5$ for numerical simulation, with the denser mesh in the vicinity of its bounding surface.

The most significant result of the asymptotic analysis is perhaps the existence of an asymptotic law $|\boldsymbol{u}| \sim P o E^{-1 / 2}$ or $E_{k i n} \sim P o^{2} E^{-1}$ at $E \ll 1$. Figure 3(a) shows, computed from the asymptotic expression (3.77), the dependence of the kinetic energy $E_{k i n}$ of the resonant flow on $E^{-1}$ for the fixed value of $\mathscr{E}=0.5$ and $P o=0.005$, while figure $3(b)$ illustrates how the kinetic energy depends on the libration frequency $\hat{\omega}$. They will be discussed in detail in $\S 4$ along with the corresponding results of direct numerical simulation.

\section{Numerical simulation}

The primary purpose of three-dimensional direct numerical simulation, which is valid for any size of $E$, is to validate the accuracy of the asymptotic solution valid only for $E \ll 1$. We shall focus on the cases with $E \ll 1$ and $|P o| \ll 1$. Local numerical methods like finite element methods are particularly suitable for non-spherical geometry. For the sake of completeness, we shall briefly discuss the finite element method, including both spatial and temporal discretization for (2.6) and (2.7), used in simulating latitudinally libration-driven flow in spheroidal cavities. The three-dimensional tetrahedralization of a spheroidal cavity in our code produces a finite element mesh that does not have pole or central numerical singularities. Moreover, the three-dimensional mesh is flexible enough to construct more nodes in the vicinity of the bounding surface of the spheroidal cavity for resolving the thin viscous boundary layer. For the simulation reported here, we have used 302968 tetrahedral elements with a total of 1152054 unknowns. A sketch of the finite element mesh for a spheroidal cavity with $\mathscr{E}=0.5$ is illustrated in figure 4 . A semi-implicit time stepping scheme is employed for the time advancement, with an implicit second-order backward differentiation formula being used for the time derivative:

$$
\left(\frac{\partial \boldsymbol{u}}{\partial t}\right)^{n+1}=\frac{3 \boldsymbol{u}^{n+1}-4 \boldsymbol{u}^{n}+\boldsymbol{u}^{n-1}}{2 \Delta t}+O\left(\Delta t^{2}\right)
$$


where $\boldsymbol{u}^{n}$ denotes $\boldsymbol{u}\left(t_{n}\right)$ with $t_{n+1}-t_{n}=\Delta t$. A second-order extrapolation is applied to the nonlinear term $\boldsymbol{u} \cdot \nabla \boldsymbol{u}$, which at $t=t_{n+1}$ can be expressed as

$$
\boldsymbol{u}^{n+1} \cdot \nabla \boldsymbol{u}^{n+1}=2\left(\boldsymbol{u}^{n} \cdot \nabla \boldsymbol{u}^{n}\right)-\left(\boldsymbol{u}^{n-1} \cdot \nabla \boldsymbol{u}^{n-1}\right)+O\left(\Delta t^{2}\right) .
$$

This semi-implicit temporal discretization of (2.6) and (2.7) gives

$$
\begin{gathered}
\frac{3 \boldsymbol{u}^{n+1}-4 \boldsymbol{u}^{n}+\boldsymbol{u}^{n-1}}{2 \Delta t}+2\left(\boldsymbol{u}^{n} \cdot \nabla \boldsymbol{u}^{n}\right)-\left(\boldsymbol{u}^{n-1} \cdot \nabla \boldsymbol{u}^{n-1}\right)+2 \hat{z} \times \boldsymbol{u}^{n+1} \\
=-\nabla p^{n+1}+E \nabla^{2} \boldsymbol{u}^{n+1}+2 P o\left[\hat{\omega}^{-1} \hat{\boldsymbol{y}} \times \boldsymbol{u}^{n+1} \cos \left(\hat{\omega} t_{n+1}\right)-\hat{\boldsymbol{x}} \times \boldsymbol{u}^{n+1} \sin \left(\hat{\omega} t_{n+1}\right)\right] \\
+P o\left[\hat{\omega} \boldsymbol{r} \times \hat{\boldsymbol{x}} \cos \left(\hat{\omega} t_{n+1}\right)+\boldsymbol{r} \times(\hat{z} \times \hat{\boldsymbol{x}}) \sin \left(\hat{\omega} t_{n+1}\right)\right], \\
\boldsymbol{\nabla} \cdot \boldsymbol{u}^{n+1}=0 .
\end{gathered}
$$

It should be pointed out that, since the Coriolis term $2 \hat{z} \times \boldsymbol{u}^{n+1}$ is primarily dominant, the two other Coriolis terms in (4.3), 2 Po $\hat{\omega}^{-1} \hat{\boldsymbol{y}} \times \boldsymbol{u}^{n+1} \cos \left(\hat{\omega} t_{n+1}\right)$ and $2 P o \hat{\boldsymbol{x}} \times \boldsymbol{u}^{n+1} \sin \left(\hat{\omega} t_{n+1}\right)$, are small and insignificant when Po $\ll 1$. There are no noticeable differences between the numerical solutions obtained with or without having the two small Coriolis terms. These equations are solved efficiently on modern parallel computers, starting from an arbitrary initial condition to find $\boldsymbol{u}^{n+1}$ and $p^{n+1}$ from given $\boldsymbol{u}^{n}$ and $\boldsymbol{u}^{n-1}$. A discussion regarding the accuracy and convergence of our spheroidal finite element code can be found in Chan, Zhang \& Liao (2010).

Consider first the librationally non-resonant flow for $\left|\hat{\omega}-2 /\left(2-\mathscr{E}^{2}\right)\right| \gg E^{1 / 2}$ at $E \ll 1$. In this case, a satisfactory agreement between the asymptotic solution and the numerical simulation is achieved. Figure 1 shows kinetic energies $E_{k i n}$ as a function of time, computed from both numerical simulation and the asymptotic expression (3.57) for $P o=0.005$ and $E=5 \times 10^{-5}$ at several different libration frequencies. It can be seen that the result from the analytical formula (3.57), which is shown by the dashed lines in figure 1, agrees quantitatively well with direct simulation without substantial noticeable differences. For example, the numerical simulation in figure 1 gives the average kinetic energy $\left(E_{\text {kin }} / P O^{2}\right)_{\text {num }}=0.164$ at $\hat{\omega}=1.35$ while the analytical formula (3.57) yields $\left(E_{\text {kin }} / P o^{2}\right)_{\text {asym }}=0.160$. Figure 2 depicts the profiles of librating flow for two different frequencies obtained from both the numerical simulation and asymptotic solution (3.53). It should be pointed out that our numerical simulation is nonlinear, representing a solution to the full equation (2.6) whilst the nonlinear term $\boldsymbol{u} \cdot \nabla \boldsymbol{u}$ is neglected in the asymptotic analysis. An excellent agreement between the numerical and asymptotic analysis is probably attributable to the fact that the interior part of the asymptotic solution (3.53) has the properties $\nabla^{2} \boldsymbol{u}=0$ and $\boldsymbol{u} \cdot \nabla \boldsymbol{u}=\boldsymbol{\nabla} \Phi$, where $\Phi$ can be absorbed into the reduced pressure. In other words, the asymptotic solution (3.53), apart from in the viscous boundary layer, does satisfy (2.6) including the term $\boldsymbol{u} \cdot \nabla \boldsymbol{u}$. There is, however, a noticeable difference in figure 2 between the asymptotic solution (3.53) and the corresponding numerical simulation, which would be attributable to the nonlinear effect in the viscous boundary layer neglected in our asymptotic analysis.

Consider now the librationally resonant flow at the libration frequency $\hat{\omega}=$ $2 /\left(2-\mathscr{E}^{2}\right)$. Our asymptotic solution (3.76) predicts that resonance with the spheroidal inertial mode $\boldsymbol{u}_{10}$ would result in an asymptotic law $|\boldsymbol{u}| \sim P o / E^{1 / 2}$ or $E_{k i n} \sim P o^{2} / E$ at $E \ll 1$. This prediction is convincingly confirmed by our direct numerical simulation performed for various values of $E$ at a fixed value of $P o$ and $\mathscr{E}$. Figure 3(a) (the solid line) shows the scaled kinetic energy, $E_{k i n} / P^{2}$, as a function of the Ekman number $E$ from direct numerical simulation. For the purpose of comparison, both the asymptotic result (the dashed line) from the formula (3.77) and the numerical 

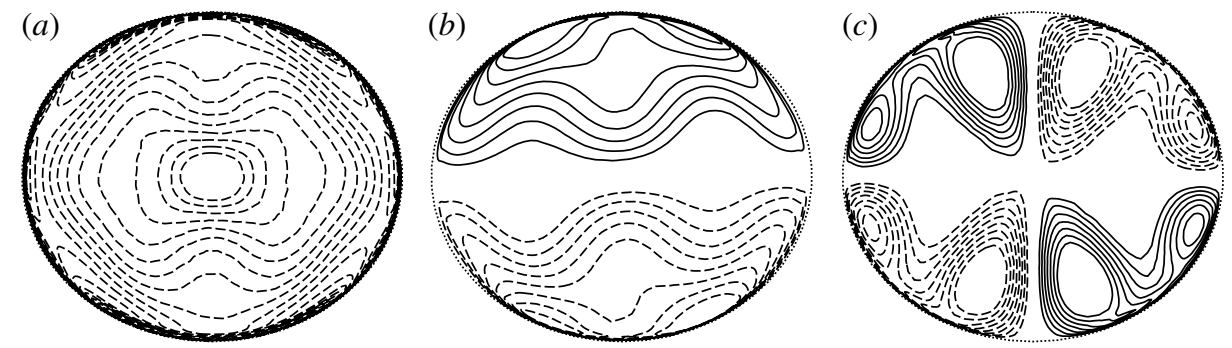

FIGURE 5. Contours of (a) $\hat{\boldsymbol{\tau}} \cdot \boldsymbol{u},(b) \hat{\boldsymbol{\phi}} \cdot \boldsymbol{u}$ and $(c) \hat{\boldsymbol{\eta}} \cdot \boldsymbol{u}$ in a meridional plane for the resonant numerical solution obtained at $P o=0.005$ and $E=5 \times 10^{-5}$ with eccentricity $\mathscr{E}=0.5$.

result are displayed in the same figure. Note that the solid line in figure $3(a)$ is based on four numerical simulations for $\mathscr{E}=0.5$ and $P o=0.005$ at Ekman number $E=10^{-2}, 10^{-3}, 10^{4}$ and $5 \times 10^{-5}$. There is a satisfactory agreement between the asymptotic solution and direct numerical simulation when $E$ is sufficiently small. For example, we obtain $\left(E_{k i n} / P o^{2}\right)_{\text {num }}=1.08$ for $E=10^{-4}$ while the asymptotic formula (3.77) gives $\left(E_{\text {kin }} / P o^{2}\right)_{\text {asym }}=1.13$. At a smaller value, $E=5 \times 10^{-5}$, the simulation gives $\left(E_{k i n} / P o^{2}\right)_{n u m}=2.28$ while the asymptotic formula (3.77) gives $\left(E_{k i n} / P o^{2}\right)_{a s y m}=2.27$, as shown in figure $3(a)$. The profile of a weakly nonlinear resonant flow in a meridional plane is displayed in figure 5 for $E=5 \times 10^{-5}$ and $P o=0.005$, indicating the existence of a strong shear in the vicinity of the bounding surface of the fluid container. In figure $3(b)$, the scaled kinetic energy is shown as a function of the libration frequency $\hat{\omega}$ for the fixed Ekman number $E=5 \times 10^{-5}$ at $\mathscr{E}=0.5$. It reveals again a satisfactory agreement between the asymptotic solutions (the solid line) and direct numerical simulations at several different frequencies (the * symbols). Our numerical simulation also confirms that the librating flow at the resonance is indeed in the form of an azimuthally, retrogradely travelling wave at the phase speed $2 /\left(2-\mathscr{E}^{2}\right)$, consistent with the asymptotic solution (3.76).

\section{Summary and remarks}

We have investigated, via both asymptotic and numerical analysis, fluid motion in a spheroidal cavity driven by latitudinal libration for an asymptotically small $E$. For the non-resonant frequency, $\left|\hat{\omega}-2 /\left(2-\mathscr{E}^{2}\right)\right| \gg O\left(E^{1 / 2}\right)$, we have derived an asymptotic solution for $E \ll 1$ that satisfies the no-slip boundary condition without making any prior assumptions about the flow structure for an arbitrary eccentricity $0<\mathscr{E}<1$. In this case, the librating flow has the typical amplitude $|\boldsymbol{u}|=O(P o)$ at $E \ll 1$ and the role of the viscous boundary layer is mainly passive for the flow to obey the no-slip condition. At the resonant frequency, $\hat{\omega}=2 /\left(2-\mathscr{E}^{2}\right)$, latitudinal libration resonates with the spheroidal inertial mode $\boldsymbol{u}_{10}$ - which represents an azimuthally retrogradely travelling wave - and the librating flow reaches a much larger amplitude $|\boldsymbol{u}|=O\left(P o / E^{1 / 2}\right)$ at $E \ll 1$. It is the viscous boundary layer that plays an essential role in determining the key property of the resonant flow. Through the viscous process, the thin boundary layer communicates with the bulk of the fluid and determines the final equilibrium of the resonant travelling wave. The strong velocity shear between the bulk fluid and the spheroidal container would provide the major source of energy dissipation. When $\mathscr{E}^{2} \ll 1$, an explicitly analytical solution is derived for the librationally resonant flow. For an oblate spheroidal cavity of arbitrary eccentricity, however, there exist two integrals in the asymptotic solution that have to be evaluated 
numerically. We also carry out direct numerical simulation, which shows a satisfactory agreement with the asymptotic solution at $E \ll 1$.

A physically significant finding in this study is the resonance of latitudinal libration with a spheroidal inertial mode when the libration frequency is close to $2 /\left(2-\mathscr{E}^{2}\right)$. It should be noted that inertial waves are ubiquitous in rotating fluid systems and may be excited and maintained by various mechanisms, such as thermal convection (e.g. Zhang 1994), precession (e.g. Lorenzani \& Tilgner 2001), a tidal effect (e.g. Le Bars et al. 2010) and differential rotation (e.g. Kelley et al. 2007). In the present study, we have revealed for the first time the resonance of an inertial wave with latitudinal libration in a spheroidal cavity that gives rise to an asymptotic law $|\boldsymbol{u}| \sim P_{o} / E^{1 / 2}$ at $E \ll 1$. The physical reason why resonance can occur in latitudinal libration with spheroidal geometry $(\mathscr{E} \neq 0)$ is actually very simple. As a consequence of spheroidal geometry, the topographic coupling between the latitudinally librating container and the interior fluid always forces fluid motion. The fluid motion would be constantly reinforced to give rise to resonance, as in the situation of a resonant pendulum, when the flow is in the form of an azimuthally travelling wave marked by the azimuthal wavenumber $m=1$ and the frequency $2 /\left(2-\mathscr{E}^{2}\right)$ such that it is always at exactly the same phase as that of latitudinal libration. By contrast, this type of resonance cannot take place in longitudinal libration because all inertial waves in spheroidal geometry always travel, prograde or retrograde, in the one direction (Zhang et al. 2004). It is hoped that the latitudinal-libration resonance in a spheroidal cavity, predicted by this asymptotic theory, will be confirmed by laboratory experiments in the future.

This new finding may have important implications for planetary magnetism. Although it is a widely accepted hypothesis that thermal or chemical buoyancy within planetary fluid cores drives planetary dynamos, the validity of this hypothesis might be questionable for certain planets and moons such as Mercury and Ganymede. In this case, an alternative mechanism, suggested by the result of this study, would be a non-thermal planetary dynamo that is driven by resonant latitudinal libration. This mechanism is possible because the two conditions for resonance - nearly synchronous rotation and small but non-zero eccentricity - are approximately met by those planets and moons. A large-amplitude flow with strong shears at $E \ll 1$ at resonance is probably susceptible to instabilities, leading to a more complicated flow, which would play a key role in the magnetism of nearly synchronous planets that are thermally or chemically non-convective. Our numerical study on how latitudinally resonant flows maintain dynamo action in a librating ellipsoid is currently under way.

Both the asymptotic and numerical solutions discussed in this paper are for weakly librating flow with a small Poincaré number $|P o| \ll 1$. When the Poincaré number becomes sufficiently large, it is anticipated that a strong mean zonal flow is likely to be generated through nonlinear interaction taking place primarily within the viscous boundary layer. The result of direct numerical simulation, focusing on strongly nonlinear flow with large values of $P o$ as well as different values of eccentricity $\mathscr{E}$, will be reported in a future paper.

\section{Acknowledgements}

K.Z. would like to thank J. M. Aurnou, F. H. Busse, J. L. Margot and J. Noir for helpful discussions. In particular, J. Noir pointed out that the term $(P o / \hat{\omega}) \cos \left(\Omega_{0} \hat{\omega} t\right) \hat{\boldsymbol{y}} \times \boldsymbol{u}$ in (2.3) is physically required because the rotation vector $\Omega_{0}$ in planetary latitudinal libration is fixed in the inertial frame. K.Z. is supported by UK NERC, STFC and Leverhulme Trust grants. Part of this work (K.Z.) was 
carried out at, and supported by, the Institute of Mathematical Sciences, the Chinese University of Hong Kong. K.H.C. is supported by Hong Kong RGC grant/700310 and X.L. is supported by NSFC/10633030 and CAS grants. The parallel computation is supported by Shanghai Supercomputer Center and Swiss National Supercomputing Center.

\section{Appendix. Asymptotic expressions for $\mathscr{I}_{r}$ and $\mathscr{I}_{i}$}

Consider first the simpler integral $\mathscr{I}_{r}$. Since the integrand is singular at $\tau_{c}=$ $-1 / \sqrt{4-\mathscr{E}^{2}}$, we may separate $\mathscr{I}_{r}$ into the two different integrals:

$$
\begin{aligned}
\mathscr{I}_{r}= & \lim _{\delta \rightarrow 0}\left\{\int_{-1}^{\tau_{c}-\delta} \frac{\left(1-\mathscr{E}^{2}+\mathscr{E}^{2} \tau^{2}\right)^{3 / 4}\left(-\tau+\sqrt{1-\mathscr{E}^{2}+\mathscr{E}^{2} \tau^{2}}\right)\left(1-2 \tau^{2}+\tau \sqrt{1-\mathscr{E}^{2}+\mathscr{E}^{2} \tau^{2}}\right)}{\left|\left(2-\mathscr{E}^{2}\right) \tau+\tau \sqrt{1-\mathscr{E}^{2}+\mathscr{E}^{2} \tau^{2}}\right|^{1 / 2} \mathrm{~d} \tau}\right. \\
& \left.+\int_{\tau_{c}+\delta}^{+1} \frac{\left(1-\mathscr{E}^{2}+\mathscr{E}^{2} \tau^{2}\right)^{3 / 4}\left(-\tau+\sqrt{1-\mathscr{E}^{2}+\mathscr{E}^{2} \tau^{2}}\right)\left(1-2 \tau^{2}+\tau \sqrt{1-\mathscr{E}^{2}+\mathscr{E}^{2} \tau^{2}}\right)}{\mid\left(2-\mathscr{E}^{2}\right) \tau+\tau{\sqrt{1-\mathscr{E}^{2}+\mathscr{E}^{2} \tau^{2}}}^{1 / 2}} \mathrm{~d} \tau\right\} .
\end{aligned}
$$

We shall assume that $\mathscr{E}^{2}$ is small but fixed. The root of the complication stems from the fact that the upper or lower limit of integration is also a function of the expansion parameter $\mathscr{E}^{2}$. Expanding both the limits and integrand in terms of $\mathscr{E}^{2}$ yields the four integrals:

$$
\begin{aligned}
\mathscr{I}_{r}= & \lim _{\delta \rightarrow 0}\left\{\int_{-1}^{-1 / 2-\delta} \frac{(1-\tau)^{2}(1+2 \tau)}{[-(1+2 \tau)]^{1 / 2}} \mathrm{~d} \tau+\int_{-1 / 2+\delta}^{1} \frac{(1-\tau)^{2}(1+2 \tau)}{(1+2 \tau)^{1 / 2}} \mathrm{~d} \tau\right. \\
& -\mathscr{E}^{2}\left[\int_{-1}^{-1 / 2-\delta} \frac{(1-\tau)^{2}\left(2+6 \tau+2 \tau^{2}-3 \tau^{3}\right)}{2[-(1+2 \tau)]^{1 / 2}} \mathrm{~d} \tau\right. \\
& \left.\left.+\int_{-1 / 2+\delta}^{1} \frac{(1-\tau)^{2}\left(2+6 \tau+2 \tau^{2}-3 \tau^{3}\right)}{2(1+2 \tau)^{1 / 2}} \mathrm{~d} \tau\right]\right\}+O\left(\mathscr{E}^{4}\right) .
\end{aligned}
$$

Each of the above four integrals is finite and can be readily evaluated, giving rise to

$$
\mathscr{I}_{r}=-\frac{2}{35}(19-9 \sqrt{3})-\mathscr{E}^{2}\left[\frac{4}{55}\left(\frac{1}{9}+6 \sqrt{3}\right)\right]+O\left(\mathscr{E}^{4}\right) .
$$

This asymptotic expression provides a reasonably accurate approximation of $\mathscr{I}_{r}$ when $\mathscr{E}^{2} \ll 1$. For example, the numerical integration of $\mathscr{I}_{r}$ at $\mathscr{E}=0.25$ gives $\left(\mathscr{I}_{r}\right)_{\text {num }}=-0.2436$ while the asymptotic formula yields $\left(\mathscr{I}_{r}\right)_{\text {asym }}=-0.2427$.

The second integral $\mathscr{I}_{i}$ is slightly more complicated because of the $3 / 2$ power in the denominator of the integrand. Similar to the previous case, we can also separate $\mathscr{I}_{i}$ into the two different integrals:

$$
\begin{aligned}
\mathscr{I}_{i}= & \lim _{\delta \rightarrow 0}\left\{\int_{-1}^{\tau_{c}-\delta} \frac{\left(1-\mathscr{E}^{2}+\mathscr{E}^{2} \tau^{2}\right)^{3 / 4}\left(-\tau+\sqrt{1-\mathscr{E}^{2}+\mathscr{E}^{2} \tau^{2}}\right)\left(1-2 \tau^{2}+\tau \sqrt{1-\mathscr{E}^{2}+\mathscr{E}^{2} \tau^{2}}\right)}{\left|\left(2-\mathscr{E}^{2}\right) \tau+\tau \sqrt{1-\mathscr{E}^{2}+\mathscr{E}^{2} \tau^{2}}\right|^{3 / 2}} \mathrm{~d} \tau\right. \\
& \left.+\int_{\tau_{c}+\delta}^{+1} \frac{\left(1-\mathscr{E}^{2}+\mathscr{E}^{2} \tau^{2}\right)^{3 / 4}\left(-\tau+\sqrt{1-\mathscr{E}^{2}+\mathscr{E}^{2} \tau^{2}}\right)\left(1-2 \tau^{2}+\tau \sqrt{1-\mathscr{E}^{2}+\mathscr{E}^{2} \tau^{2}}\right)}{\left|\left(2-\mathscr{E}^{2}\right) \tau+\tau \sqrt{1-\mathscr{E}^{2}+\mathscr{E}^{2} \tau^{2}}\right|^{3 / 2}} \mathrm{~d} \tau\right\} .
\end{aligned}
$$


Expanding both the integrand and limits in term of the expansion parameter $\mathscr{E}^{2}$ yields

$$
\begin{aligned}
\mathscr{I}_{i}= & \lim _{\delta \rightarrow 0}\left\{-\int_{-1}^{-1 / 2-\delta} \frac{(1-\tau)^{2}(1+2 \tau)}{[-(1+2 \tau)]^{3 / 2}} \mathrm{~d} \tau-\int_{-1 / 2+\delta}^{1} \frac{(1-\tau)^{2}(1+2 \tau)}{(1+2 \tau)^{3 / 2}} \mathrm{~d} \tau\right. \\
& +\mathscr{E}^{2}\left[\int_{-1}^{-1 / 2-\delta} \frac{(1-\tau)^{2}\left(1+4 \tau+3 \tau^{2}-3 \tau^{3}\right)}{2[-(1+2 \tau)]^{3 / 2}} \mathrm{~d} \tau\right. \\
& +\left[\frac{(1-\tau)^{2}(1+2 \tau)}{16[-(1+2 \tau)]^{3 / 2}}\right]_{\tau=-1 / 2-\delta}-\left[\frac{(1-\tau)^{2}(1+2 \tau)}{16(1+2 \tau)^{3 / 2}}\right]_{\tau=-1 / 2+\delta} \\
& \left.\left.+\int_{-1 / 2+\delta}^{1} \frac{(1-\tau)^{2}\left(1+4 \tau+3 \tau^{2}-3 \tau^{3}\right)}{2(1+2 \tau)^{3 / 2}} \mathrm{~d} \tau\right]\right\}+O\left(\mathscr{E}^{4}\right) .
\end{aligned}
$$

Whilst the two leading-order integrals can be directly evaluated, every term in the order $\mathscr{E}^{2}$ diverges and must be treated carefully. After carrying out the relevant integration, we obtain the following limits:

$$
\begin{aligned}
\mathscr{I}_{i}= & \frac{2}{5}(7-3 \sqrt{3})+\mathscr{E}^{2} \lim _{\delta \rightarrow 0}\left\{\left[\frac{81-24 \tau-93 \tau^{2}+233 \tau^{3}-160 \tau^{4}+35 \tau^{5}}{210[-(1+2 \tau)]^{1 / 2}}\right]_{-1}^{-1 / 2-\delta}\right. \\
& +\left[\frac{(1-\tau)^{2}(1+2 \tau)}{16[-(1+2 \tau)]^{3 / 2}}\right]_{\tau=-1 / 2-\delta}-\left[\frac{(1-\tau)^{2}(1+2 \tau)}{16(1+2 \tau)^{3 / 2}}\right]_{\tau=-1 / 2+\delta} \\
& \left.-\left[\frac{81-24 \tau-93 \tau^{2}+233 \tau^{3}-160 \tau^{4}+35 \tau^{5}}{210(1+2 \tau)^{1 / 2}}\right]_{-1 / 2+\delta}^{1}\right\}+O\left(\mathscr{E}^{4}\right) . \\
= & \frac{2}{5}(7-3 \sqrt{3})+\mathscr{E}^{2}\left[\frac{4}{105}(52-3 \sqrt{3})\right] \\
& +\lim _{\delta \rightarrow 0}\left\{\sqrt{2 \delta}\left[-\frac{57}{32}-\frac{61 \delta^{2}}{20}-\frac{\delta^{4}}{6}\right]\right\}+O\left(\mathscr{E}^{4}\right) .
\end{aligned}
$$

Taking the limit $\delta \rightarrow 0$ gives the asymptotic expression:

$$
\mathscr{I}_{i}=\frac{2}{5}(7-3 \sqrt{3})+\mathscr{E}^{2}\left[\frac{4}{105}(52-3 \sqrt{3})\right]+O\left(\mathscr{E}^{4}\right) .
$$

This again offers a reasonably accurate approximation for small values of $\mathscr{E}^{2}$. For example, the numerical integration of $\mathscr{I}_{i}$ at $\mathscr{E}=0.25$ gives $\left(\mathscr{I}_{i}\right)_{\text {num }}=0.8413$ while the corresponding asymptotic formula yields $\left(\mathscr{I}_{i}\right)_{\text {asym }}=0.8330$.

\section{REFERENCES}

Aldridge, K. D. \& Toomre, A. 1969 Axisymmetric inertial oscillations of a fluid in a rotating spherical container. J. Fluid Mech. 37, 307-323.

BALAND, R. \& VAN Hoolst, T. 2010 Librations of the Galilean satellites: the influence of global internal liquid layers. Icarus 209, 651-664.

Busse, F. H. 1968 Steady fluid flow in a precessing spheroidal shell. J. Fluid Mech. 136, 739-751.

Busse, F. H. 2010 Mean zonal flows generated by librations of a rotating spherical cavity. J. Fluid Mech. 650, 505-512.

Calkins, M. A., Noir, J., Eldredge, J. \& Aurnou, J. M. 2010 Axisymmetric simulations of libration-driven fluid dynamics in a spherical shell geometry. Phys. Fluids 22, 086602.

Chan, K., LiaO, X. \& Zhang, K. 2011 Simulations of fluid motion in ellipsoidal planetary cores driven by longitudinal libration. Phys. Earth Planet. Inter. 187, 391-403. 
Chan, K., Zhang, K. \& LiaO, X. 2010 An EBE finite element method for simulating nonlinear flows in rotating spheroidal cavities. Intl J. Numer. Meth. Fluids 63, 395-414.

Dermott, S. F. 1979 Shapes and gravitational moments of satellites and asteroids. Icarus 37, $576-586$.

Dufey, J., Noyelles, B., Rambaux, N. \& Lemaitre, A. 2009 Latitudinal librations of Mercury with a fluid core. Icarus 203, 1-12.

Greenspan, H. P. 1968 The Theory of Rotating Fluids. Cambridge University Press.

Kelley, D., Triana, S., Zimmerman, D., Tilgner, A. \& Lathrop, D. 2007 Inertial waves driven by differential rotation in a planetary geometry. Geophys. Astrophys. Fluid Dyn. 101, 469-487.

Kong, D., Zhang, K. \& Schubert, G. 2010 Shapes of two-layer models of rotating planets. J. Geophys. Res. 115, E12003.

Le Bars, M., Lacaze, L., Le Dizes, S., Le Gal, P. \& Rieutord, M. 2010 Tidal instability in stellar and planetary binary systems. Phys. Earth Planet. Inter. 178, 48-55.

Lorenzani, S. \& Tilgner, A. 2001 Fluid instabilities in precessing spheroidal cavities. J. Fluid Mech. 447, 111-128.

Margot, J. L., Peale, S. J., Jurgens, R. F., Slade, M. A. \& Holin, I. V. 2007 Large longitude libration of Mercury reveals a molten core. Science 316, 710-714.

Noir, J., CARdin, P., JAult, D. \& MASSON, J. P. 2003 Experimental evidence of nonlinear resonance effects between retrograde precession and the tilt-over mode within a spheroid. Geophys. J. Intl 154, 407-416.

Noir, J., Hemmerlin, F., Wicht, J., BacA, S. M. \& Aurnou, J. M. 2009 An experimental and numerical study of librationally driven flow in planetary cores and subsurface oceans. Phys. Earth Planet. Inter. 173, 141-152.

Rambaux, N., Van Hoolst, T. \& Karatekin, O. 2011 Librational response of Europa, Ganymede, and Callisto with an ocean for a non-Keplerian orbit. Astron. Astrophys. 527, A118.

RIEUTORD, M. 1991 Linear theory of rotating fluids using spherical harmonics, part II, time-periodic flows. Geophys. Astrophys. Fluid Dyn. 59, 185-208.

Roberts, P. H. \& Stewartson, K. 1965 On the motion of a liquid in a spheroidal cavity of a precessing rigid body: II. Math. Proc. Camb. Phil. Soc. 61, 279-288.

Sauret, A., Cebron, D., Morize, C. \& Le Bars, M. 2010 Experimental and numerical study of mean zonal flows generated by librations of a rotating spherical cavity. J. Fluid Mech. 662, 260-268.

Tilgner, A. 1999 Driven inertial oscillations in spherical shells. Phys. Rev. E 59, 1789-1794.

Tilgner, A. \& Busse, F. H. 2001 Fluid flows in precessing spherical shells. J. Fluid Mech. 426, 387-396.

VAnyo, J. P., Wilde, P., Cardin, P. \& Olson, P. 1995 Experiments on precessing flows in the Earth's liquid core. Geophys. J. Intl 121, 136-142.

William, J. G., Boggs, D. H., Yoder, C. F., Ratcliff, J. T. \& Dickey, J. O. 2001 Lunar rotational dissipation in solid body and molten core. J. Geophys. Res. 106, 27933-27968.

Wu, C. C. \& Roberts, P. H. 2009 On a dynamo driven by topographic precession. Geophys. Astrophys. Fluid Dyn. 103, 467-501.

ZHANG, K. 1994 On coupling between the Poincare equation and the heat equation. J. Fluid Mech. 268, 211-229.

ZhANG, K., CHAN, K. \& LiAO, X. 2011 On fluid motion in librating ellipsoids with moderate equatorial eccentricity. J. Fluid Mech. 673, 468-479.

ZHANG, K., LiAO, X. \& EARNSHAW, P. 2004 On inertial waves and oscillations in a rapidly rotating fluid spheroid. J. Fluid Mech. 504, 1-40. 